\section{APR-246 induces early cell death by ferroptosis in acute myeloid leukemia}

\author{
Rudy Birsen, ${ }^{1,2}$ Clement Larrue, ${ }^{3}$ Justine Decroocq, ${ }^{1,2}$ Natacha Johnson, ${ }^{1}$ \\ Nathan Guiraud, ${ }^{4,5}$ Mathilde Gotanegre, ${ }^{4,5}$ Lilia Cantero-Aguilar, ${ }^{1}$ \\ Eric Grignano, ${ }^{1}$ Tony Huynh, ${ }^{1}$ Michaela Fontenay, ${ }^{1,6}$ Olivier Kosmider, ${ }^{1,6}$ \\ Patrick Mayeux, ${ }^{1}$ Nicolas Chapuis, ${ }^{1,6}$ Jean Emmanuel Sarry, ${ }^{4}$ \\ Jerome Tamburini, ${ }^{1,2,3}$ and Didier Bouscary ${ }^{1,2}$
}

${ }^{1}$ Université de Paris, Institut Cochin, CNRS UMR8104, INSERM U1016, Paris, France; ${ }^{2}$ Assistance Publique-Hôpitaux de Paris, Centre-Université de Paris, Service d'Hématologie Clinique, Hôpital Cochin, Paris, France; ${ }^{3}$ Translational Research Center in Onco-Hematology, Faculty of Medicine, University of Geneva, Geneva, Switzerland; ${ }^{4}$ Centre de Recherches en Cancérologie de Toulouse, UMR1037, INSERM, Equipe Labellisée LIGUE 2018, Toulouse, France; ${ }^{5}$ Université de Toulouse, Institut National des Sciences Appliquées de Toulouse, INSERM, Toulouse, France and ${ }^{6}$ Assistance PubliqueHôpitaux de Paris, Centre-Université de Paris, Service d'Hématologie Biologique, Hôpital Cochin, Paris, France

\section{ABSTRACT}

A PR-246 is a promising new therapeutic agent that targets p53 mutated proteins in myelodysplastic syndromes and in acute myeloid leukemia (AML). APR-246 reactivates the transcriptional activity of p53 mutants by facilitating their binding to DNA target sites. Recent studies in solid cancers have found that APR-246 can also induce p53-independent cell death. In this study, we demonstrate that AML cell death occurring early after APR-246 exposure is suppressed by iron chelators, lipophilic antioxidants and inhibitors of lipid peroxidation, and correlates with the accumulation of markers of lipid peroxidation, thus fulfilling the definition of ferroptosis, a recently described cell death process. The capacity of AML cells to detoxify lipid peroxides by increasing their cystine uptake to maintain major antioxidant molecule glutathione biosynthesis after exposure to APR-246 may be a key determinant of sensitivity to this compound. The association of APR-246 with induction of ferroptosis (either by pharmacological compounds, or genetic inactivation of SLC7A11 or GPX4) had a synergistic effect on the promotion of cell death, both in vivo and ex vivo.

\section{Introduction}

Acute myeloid leukemias (AML) are highly heterogeneous diseases with a constant activation of oncogenic signaling. ${ }^{1}$ Recent years have witnessed major breakthroughs in their treatment with the approval of midostaurin, venetoclax and IDH mutant inhibitors. ${ }^{2-5}$ However, AML has a poor prognosis and there is still an urgent need for new treatments. APR-246, also known as PRIMA-1MET, is a promising new therapeutic agent that targets TP53 mutated cancers. ${ }^{6-8}$ This compound is being evaluated in AML and myelodysplastic syndromes (MDS) with TP53 mutations and appears to be highly effective against this poor prognosis disease..$^{8-11}$ Mechanistically, APR-246 is converted to a reactive product (methylene quinuclidinone, MQ) that reacts with nucleophiles and thus alkylates thiol groups in proteins. ${ }^{12}$ APR-246 reactivates the transcriptional activity of p53 mutants by facilitating their binding to DNA target sites. Specific cysteines in the core domain of mutant p53 proteins are critical targets for their reactivation by APR-246/MO. ${ }^{13}$ APR-246 also triggers p53-independent cell death mechanisms. ${ }^{14,15}$ Accordingly, using esophageal cancer as a model, it has been shown that APR-246 causes a decrease in glutathione (GSH) content resulting in an increased amount of reactive oxygen species (ROS) and of lipid peroxides in particular. ${ }^{16}$ In this report, we investigated the mechanisms of cell death induced by APR-246 in AML and we demonstrated that early cell death in AML is due to ferroptosis induction.
Ferrata Storti Foundation

\section{Correspondence:}

RUDY BIRSEN

rudy.birsen@inserm.fr

DIDIER BOUSCARY

didier.bouscary@aphp.fr

Received: May 18, 2020.

Accepted: December 28, 2020.

Pre-published: January 7, 2021.

https://doi.org/10.3324/haematol.2020.259531

(c)2022 Ferrata Storti Foundation

Material published in Haematologica is covered by copyright. All rights are reserved to the Ferrata Storti Foundation. Use of published material is allowed under the following terms and conditions:

https://creativecommons.org/licenses/by-nc/4.0/legalcode. Copies of published material are allowed for personal or internal use. Sharing published material for non-commercial purposes is subject to the following conditions:

https://creativecommons.org/licenses/by-nc/4.0/legalcode, sect. 3. Reproducing and sharing published material for commercial purposes is not allowed without permission in writing from the publisher. 


\section{Methods}

\section{Cell lines and reagents}

HL60, MOLM14, SET2, MV4-11, OCI-AML2, OCI-AML3, K562, THP1, UT7-EPO, SKM1, NB4 and KASUMI AML cell lines were used. Patients provided written informed consent in accordance with the Declaration of Helsinki. Bone marrow (BM) samples were obtained from five patients with newly diagnosed AML (characteristics provided in the Online Supplementary Table S1). Cells were cultured in RPMI with glutamine (Gibco61870, Life Technologies ${ }^{\circledR}$ Saint Aubin, France) supplemented with $10 \%$ fetal bovine serum (FBS) and $4 \mathrm{mM}$ glutamine. All AML cell lines were certified using their microsatellite identity (characteristics provided in Online Supplementary Table S2). Ferrostatin-1, necrostatin-1, necrostatin-1S, necrosulfonamide, OVD-OPH, APR-246 for the in vitro study, erastin and RSL3 were sourced from Selleckchem (Houston, TX). Chloroquine and doxycycline were obtained from Sigma-Aldrich (Saint-Louis, MO). FINO2 was purchased from Cayman Chemicals (Ann Arbor, MI). The APR-246 reagent used in the in vivo study was provided by APREA therapeutics (Solna, Sweden).

\section{Constructs}

Inducible short hairpin RNA (shRNA) targeting SLC7A11 or GPX4 were constructed as previously described ${ }^{17}$ using the following sequences: SLC7A11\#2, GCTGAATTGGGAACAACTATA; SLC7A11\#3, GCAGTTGCTGGGCTGATTTAT; GPX4\#1, GTGAGGCAAGACCGAAGTAAA; GPX4\#2, CTACAACGTCAAATTCGATAT.

\section{Lentivirus production and acute myeloid leukemia cell line infection}

293-T packaging cells were used to produce lentiviral constructs through co-transfection with plasmids encoding lentiviral proteins. Supernatants were collected and ultracentrifuged for 48 hours (h) after transfection over two consecutive days and subsequently stored at $-80^{\circ} \mathrm{C}$. AML cell lines were plated at $2 \times 10^{6} / \mathrm{mL}$ and 2-10 $\mu \mathrm{L}$ aliquots of lentiviral supernatants were added for $3 \mathrm{~h}$. Cells were then grown in $10 \%$ fetal calf serum medium and further selected with puromycin. For shRNA induction, $200 \mu \mathrm{g} / \mathrm{mL}$ doxycycline was added to the culture medium.

\section{Flow cytometry-based assay}

Data acquisition and data analysis were conducted at the Cochin Cytometry and Immunobiology Facility. For glutathione measurements using monochlorobimane (MCB; Thermofischer; Waltham, MA), $2 \times 10^{5}$ cells were labeled with $40 \mu \mathrm{M}$ MCB in 1 $\mathrm{mL}$ of warm complete medium for 20 minutes (min) in a tissue culture incubator $\left(37^{\circ} \mathrm{C}, 5 \% \mathrm{CO}_{2}\right)$ in the dark. The reaction was terminated using $1 \mathrm{~mL}$ of cold complete medium, followed by centrifugation $(200 \mathrm{xg}, 1 \mathrm{~min})$. The pelleted cells were then resuspended in $0.5 \mathrm{~mL}$ of cold complete medium and placed on ice in the dark until analysis by flow cytometry (FCM). The MCBGSH signal was detected using a $355 \mathrm{~nm}$ laser through a 450/50 $\mathrm{nm}$ filter. FCM data were collected using a BD Fortessa flow cytometer with DIVA software. 10,000 events were recorded for analysis. Data analysis was then carried out with KALUZA software. For lipid peroxide production measurements using C11BODIPY (581/591) (2 $\mu \mathrm{M})$ (Thermofischer, Waltham, MA), $2 \times 10^{5}$ cells were labeled with C11-BODIPY in $1 \mathrm{~mL}$ of warm complete medium for $10 \mathrm{~min}$ in a tissue culture incubator $\left(37^{\circ} \mathrm{C}\right.$, $\left.5 \% \mathrm{CO}_{2}\right)$ in the dark. Cells were then washed twice and resuspended in $200 \mu \mathrm{L}$ of fresh PBS. For cystine uptake measurements using BioTracker Cystine-FITC Live Cell Dye (5 $\mu \mathrm{M})$ (Thermofischer, Waltham, MA), $2 \times 10^{5}$ cells were labeled with
Biotracker cystine in $1 \mathrm{~mL}$ of warm complete medium for 120 minutes in a tissue culture incubator $\left(37^{\circ} \mathrm{C}, 5 \% \mathrm{CO}_{2}\right)$ in the dark. FCM data were collected using a C6 Accuri flow cytometer (Becton Dickinson, Le Pont de Claix, France) with CFlow Plus software. 10,000 events were captured for subsequent analysis with CFlow Plus software (Becton Dickinson, Le Pont de Claix, France).

\section{Western blotting}

Whole-cell extraction and western blotting were performed as previously described. ${ }^{17}$ Anti-GPX4 antibody was purchased from Proteintech (Manchester, UK). Anti-PARP, caspase 8, caspase 3, cleaved caspase 3, MLKL, pMLKL, p53 and SLC7A11 antibodies were sourced from Cell Signaling Technology (Danvers, MA, USA).

\section{Viability assay}

AML cells were plated at $20 \times 10^{4} / \mathrm{mL}$ in $100 \mu \mathrm{l}$ of $10 \%$ FBSsupplemented RPMI prior to the addition of compounds. Cells were cultured in the presence of the test compounds for 24 to 48 $\mathrm{h}$ at $37^{\circ} \mathrm{C}$. Viability was quantified using the fluorescence based Uptiblue assay (Interchim, Montluçon, France). Uptiblue was added to each well in $10 \mu \mathrm{L}$ aliquots. Fluorescence was then measured with a Typhoon FLA9500 scanner (GE Healthcare; IL). Fluorescence values were normalized to dimethyl sulfoxide (DMSO)-treated controls for each AML cell line. Half maximal inhibitory concentration $\left(\mathrm{IC}_{50}\right)$ values were calculated using a four parameter non-linear regression curve with Graph Pad Prism v8 (GraphPad, La Jolla, CA, USA). For primary AML cells, viability was assayed by FCM analysis using forward scatter (FSC) versus side scatter (SSC).

\section{Measurement of synergistic effects}

Cell viability was calculated for every dose combination of APR-246 and ferroptosis inducer using the Synergy Finder webtool (https://synergyfinder.fimm.fi) and compared to each agent alone. Calculations were based on the ZIP model. ${ }^{18}$

\section{Tumor xenografts in NOD/SCID IL-2 receptor $\gamma$-chain-null mice}

Xenograft tumors were generated by randomly injecting $1 \times 10^{6}$ MOLM14 shCTRL or shSLC7A11 cells into the tail veins of NOD/SCID IL-2 receptor $\gamma$-chain-null mice (NSG) aged 6-9 weeks. Fourteen days after injection, doxycycline $(200 \mu \mathrm{g} / \mathrm{mL})$ and sucrose ( $1 \%$ weight:volume) were added to the drinking water of these animals. After 3 days, the mice were randomly treated with a daily intraperitoneal injection of APR-246 (100 $\mathrm{mg} / \mathrm{kg}$ ) or vehicle (phosphate-buffered saline [PBS]) for 4 days. All experiments were conducted in accordance with the guidelines of the Association for Assessment and Accreditation of Laboratory Animal Care International. Animals were used in accordance with a protocol reviewed and approved by the Institutional Animal Care and Use Committee of Région MidiPyrénées (France). BM (mixed from tibias and femurs) were dissected and flushed in Hanks balanced salt solution with $1 \%$ FBS. MNC from BM were labeled with PE-conjugated anti-hCD33, PerCP-Cy5.5-conjugated anti-mCD45.1 and APC-conjugated anti-hCD45 (all antibodies from Becton Dickinson, BD) to determine the fraction of human blasts ( $\mathrm{hCD} 45^{+} \mathrm{mCD} 45.1-\mathrm{hCD}^{+} 3^{+}$ cells) using FCM. Acquisition of data was performed on a CytoFLEX (Beckman Coulter) flow cytometer with CytExpert software. The number of AML cells in the BM was determined using CountBright beads (Invitrogen, CA, USA) in accordance with the manufacturer's protocol. Data analysis was performed with flowJo software. 
A

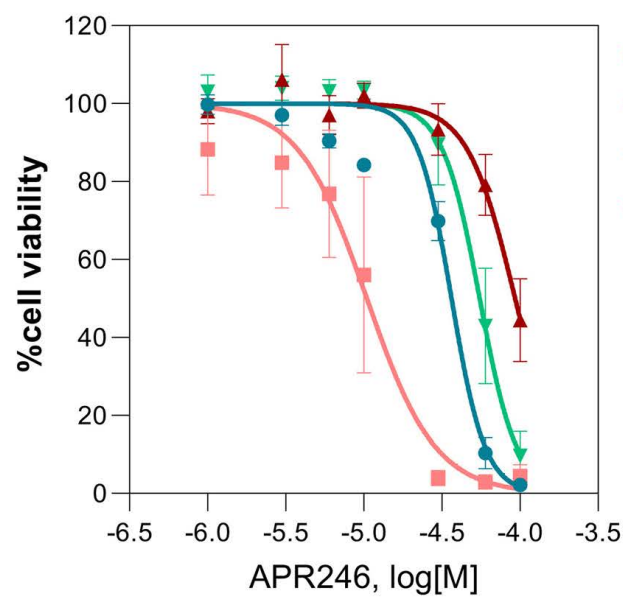

B

AML cell lines

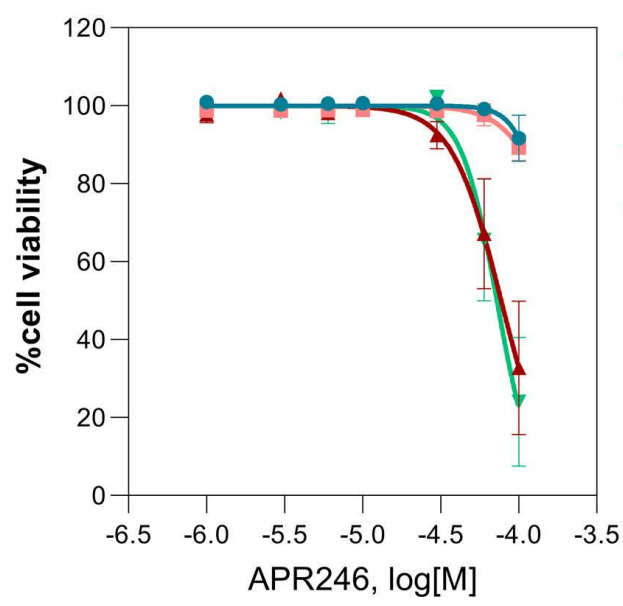

C

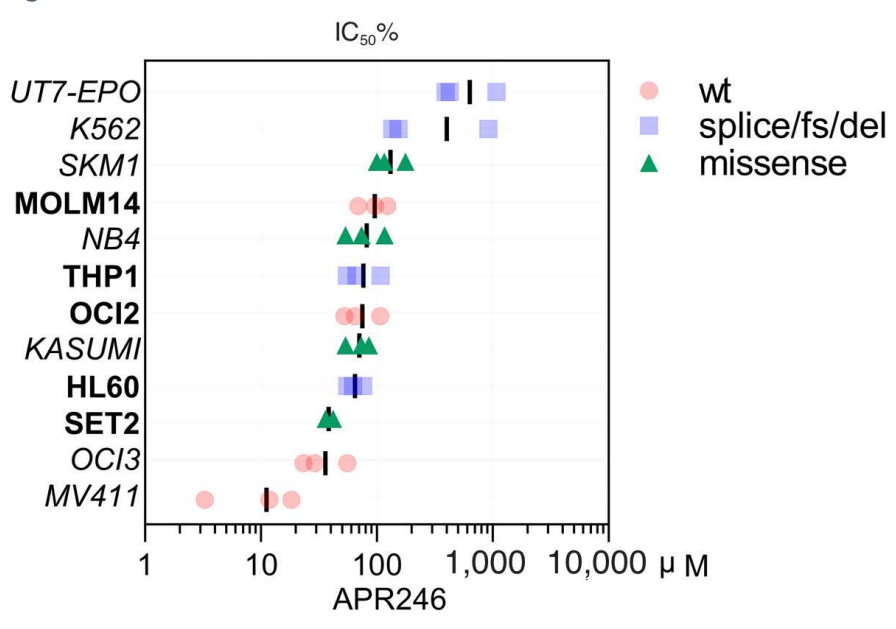

AML cell lines

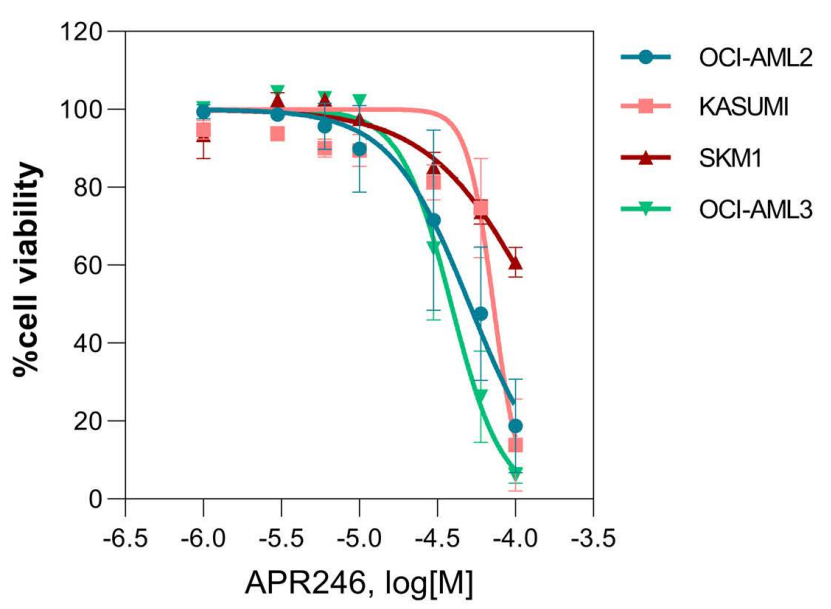

Primary AML Cells

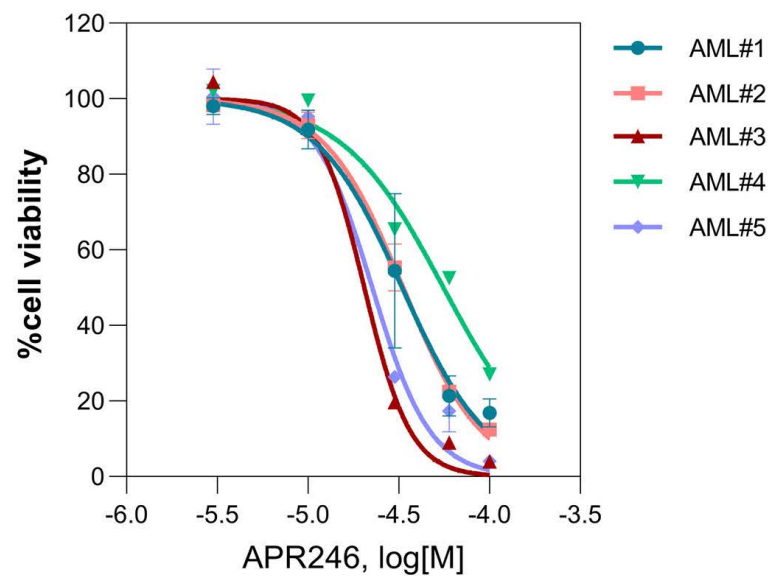

Figure 1. APR-246 induces cell death in acute myeloid leukemia cells irrespective of their TP53 mutational status. (A) Viability curves for the indicated cells at 24 hours (h) post APR-246 treatment. Error bars, \pm standard deviation. (B) Viability curves for the indicated primary acute myeloid leukemia (AML) cells at $24 \mathrm{~h}$ post APR-246 treatment. Patient characteristics are provided in the Online Supplementary Table S1. (C) Half maximal inhibitory concentration percentage (IC $\mathrm{C}_{50}$ ) of APR246 treatment for $24 \mathrm{~h}$ across a panel of AML cell lines based on cell viability $(n=3)$. In our subsequent experiments, we selected five AML cell lines sensitive to APR246 in these concentration ranges, with or without TP53 mutations (highlighted in bold font). wt: wild-type; fs: frameshift; del: deletion. 

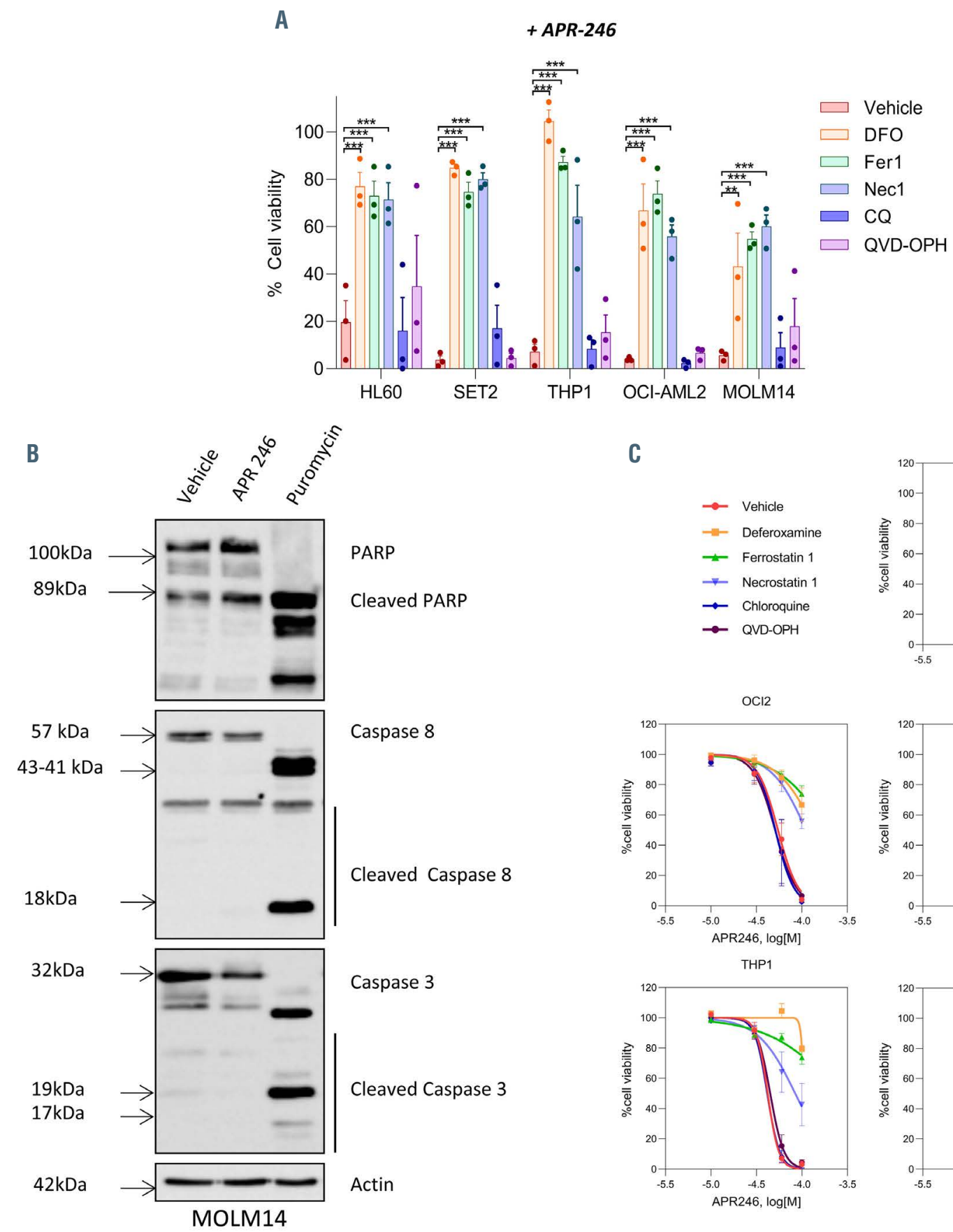

C
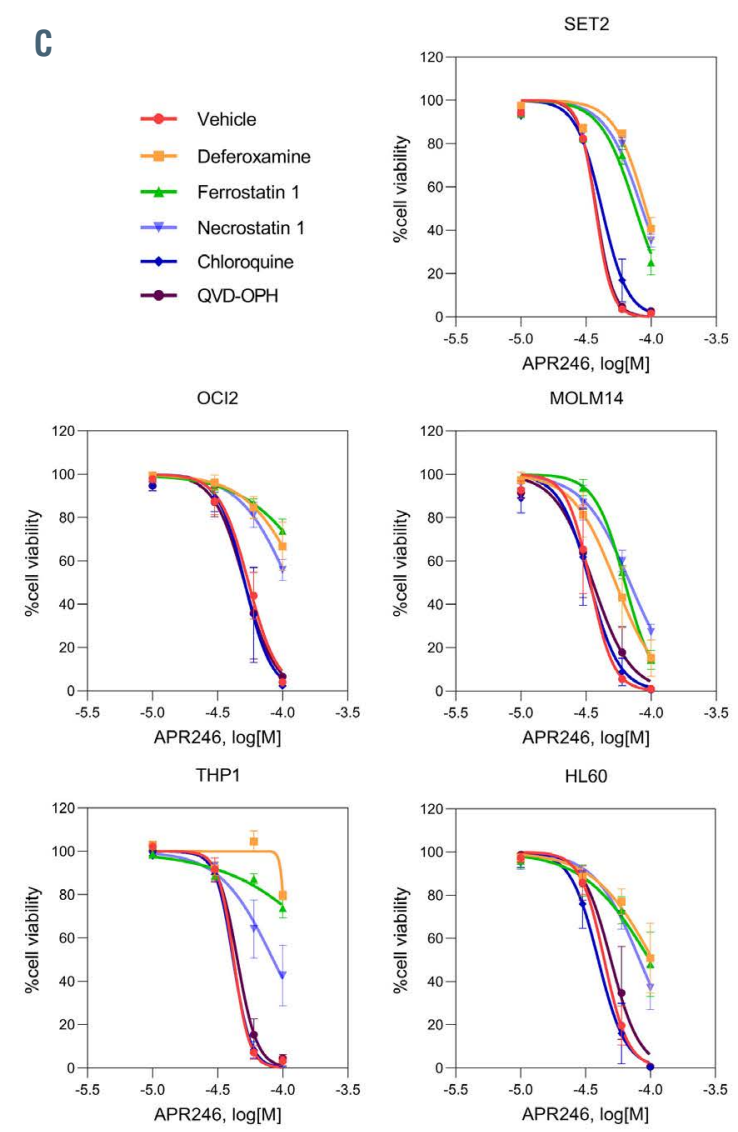

。

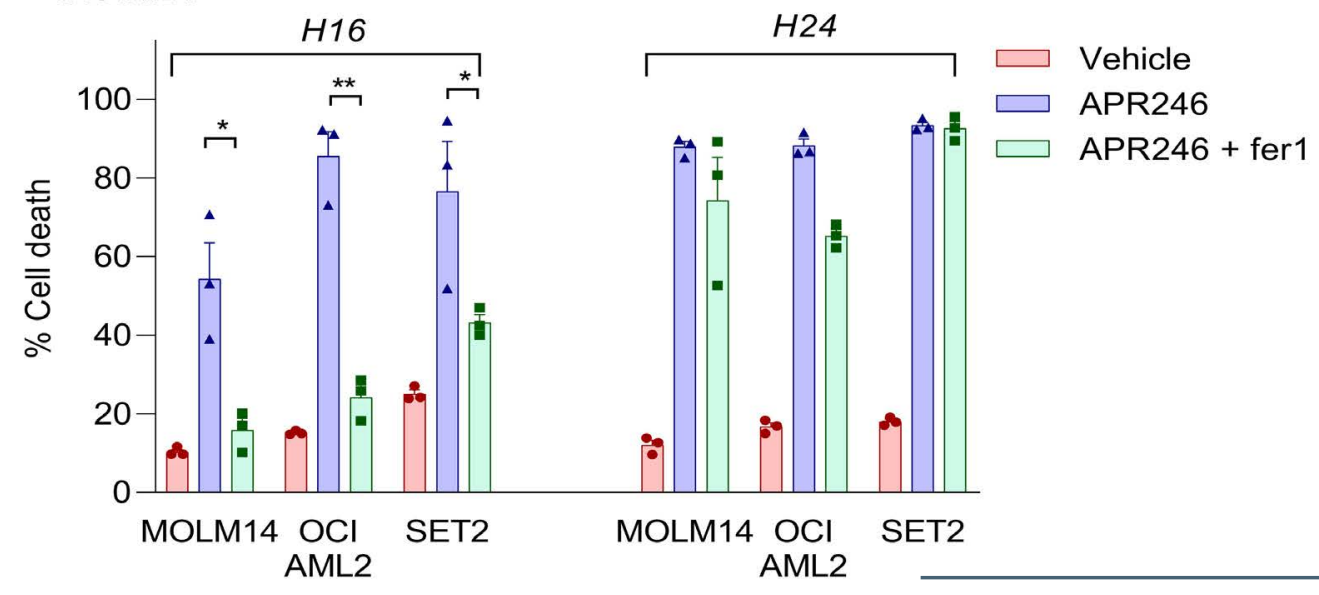


Figure 2. APR-246 induces ferroptosis in acute myeloid leukemia cells. (A) Cell viability (\%) for the indicated cells at 16 hours (h) post-APR-246 treatment (60 $\mu$ M) with or without ferrostatin-1 $(10 \mu \mathrm{M})$, deferoxamine (DFO) $(100 \mu \mathrm{M})$, necrostatin-1 $(20 \mu \mathrm{M})$, chloroquine $(20 \mu \mathrm{M})$ or QVD-OPH $(25 \mu \mathrm{M})(\mathrm{n}=3)$. Error bars \pm standard error of the mean [SEM]. All compounds were added $2 \mathrm{~h}(\mathrm{~h})$ prior to APR-246 in the medium. Statistics, 2-way ANOVA; $* P<0.05, * * P<0.01, * * * P<0.0001$. (B) Immunoblotting analysis of PARP, caspase 8 and caspase 3 in MOLM-14 cells treated for $16 \mathrm{~h}$ with dimethyl sulfoxide (DMSO), APR-246 (60 $\mu$ M) or puromycin ( 1 $\mu \mathrm{g} / \mathrm{mL})$. $\beta$-actin was used as a loading control $(\mathrm{n}=2)$. (C) Viability curves for the indicated cells at $16 \mathrm{~h}$ post APR-246 treatment with or without ferrostatin-1 $(10 \mu \mathrm{M})$, DFO $(100 \mu \mathrm{M})$, necrostatin-1 $(20 \mu \mathrm{M})$, chloroquine $(20 \mu \mathrm{M})$ or QVD-OPH $(25 \mu \mathrm{M})(\mathrm{n}=3)$. Error bars \pm SEM. (d) Cell death $(\%)$ of the indicated cells at $16 \mathrm{~h}$ and $24 \mathrm{~h}$ post-APR-246 treatment $(50 \mu \mathrm{M})$ with or without ferrostatin-1 $(10 \mu \mathrm{M})(\mathrm{n}=3)$. Error bars \pm standard deviation.

A
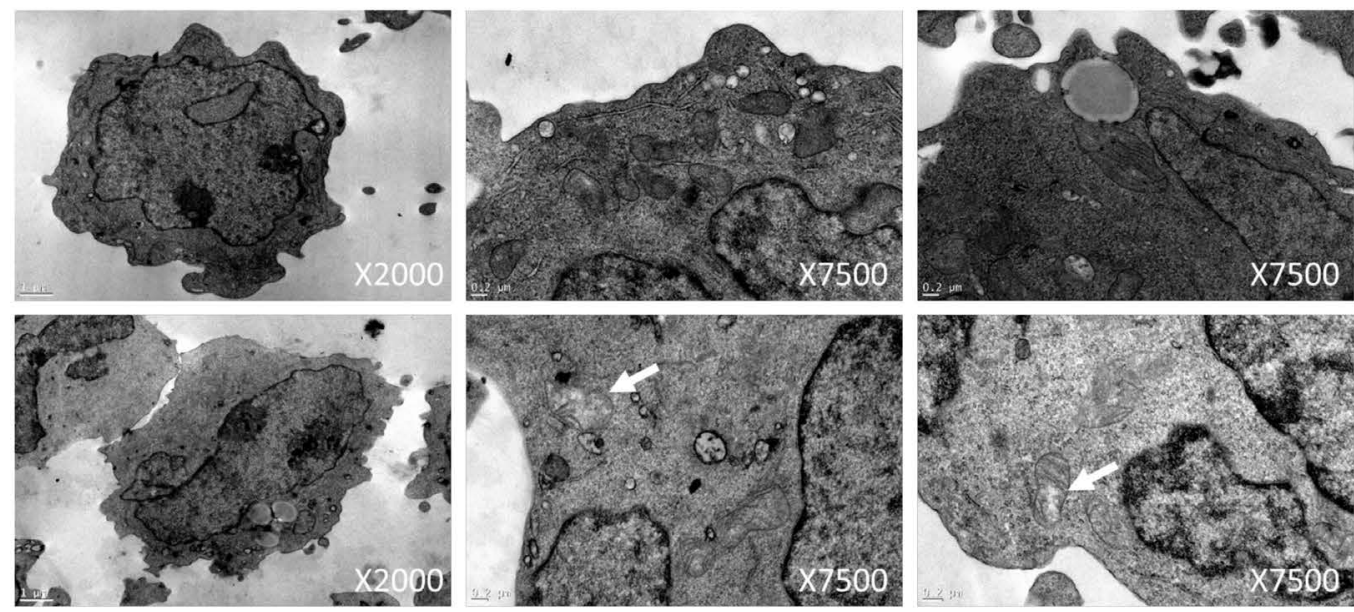

B

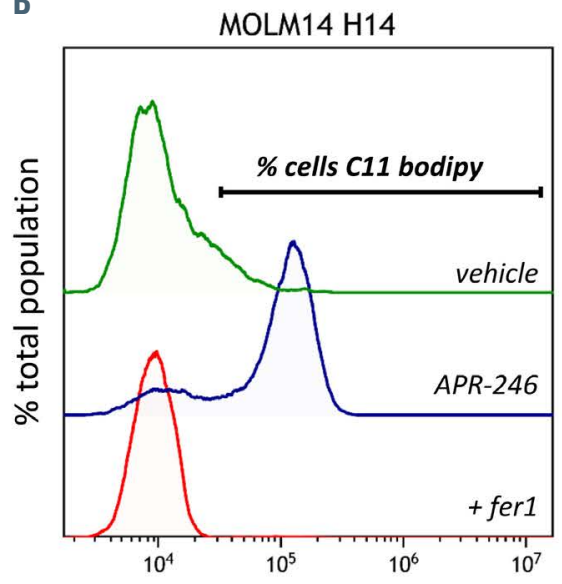

C11 BODIPY-FITC (lipid peroxydation)

C

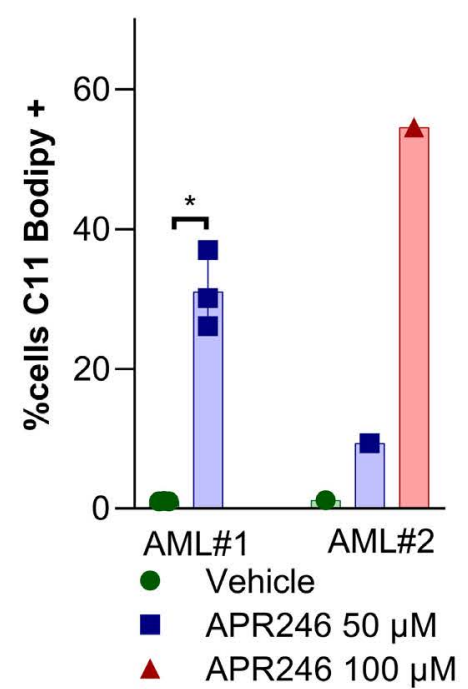

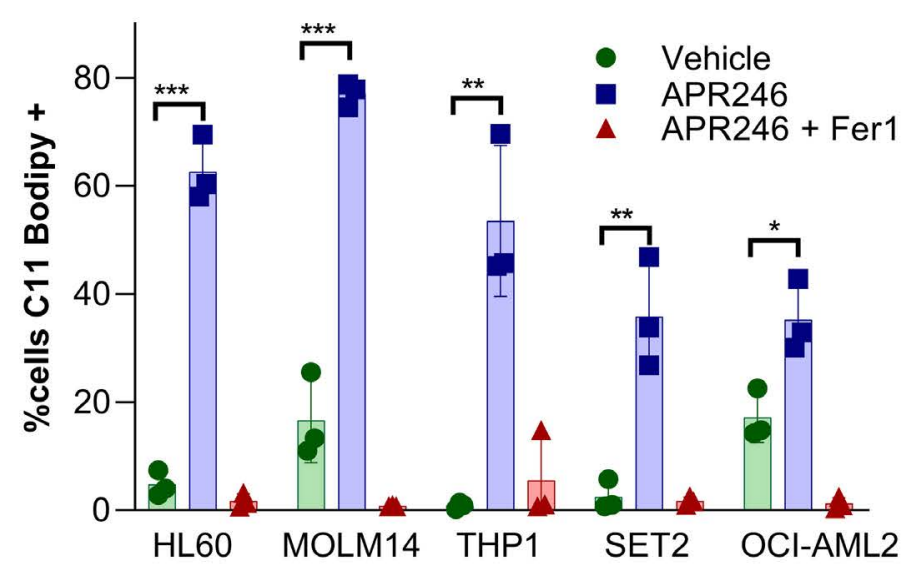

Figure 3. APR-246 induces ferroptosis in acute myeloid leukemia cells. (A) Electron microscopy analysis of MOLM-14 cells treated with or without APR-246 (60 $\mu \mathrm{M}, \mathrm{H} 16)$. The white arrowhead indicates a mitochondrion showing membrane rupture and reduced cristae. (B and C) Detection of lipid peroxidation using C11-BODIPY and flow cytometry (FCM) at 14 hours post APR-246 treatment in acute myeloid leukemia (AML) cell lines (B) and in primary AML cells (C). APR-246 was used at a $100 \mu \mathrm{M}$ concentration for MOLM-14 and $50 \mu \mathrm{M}$ for other AML cell lines. Left panels show representative FCM quantification $(n=3)$. Error bars \pm standard deviation. 
Erastin

A

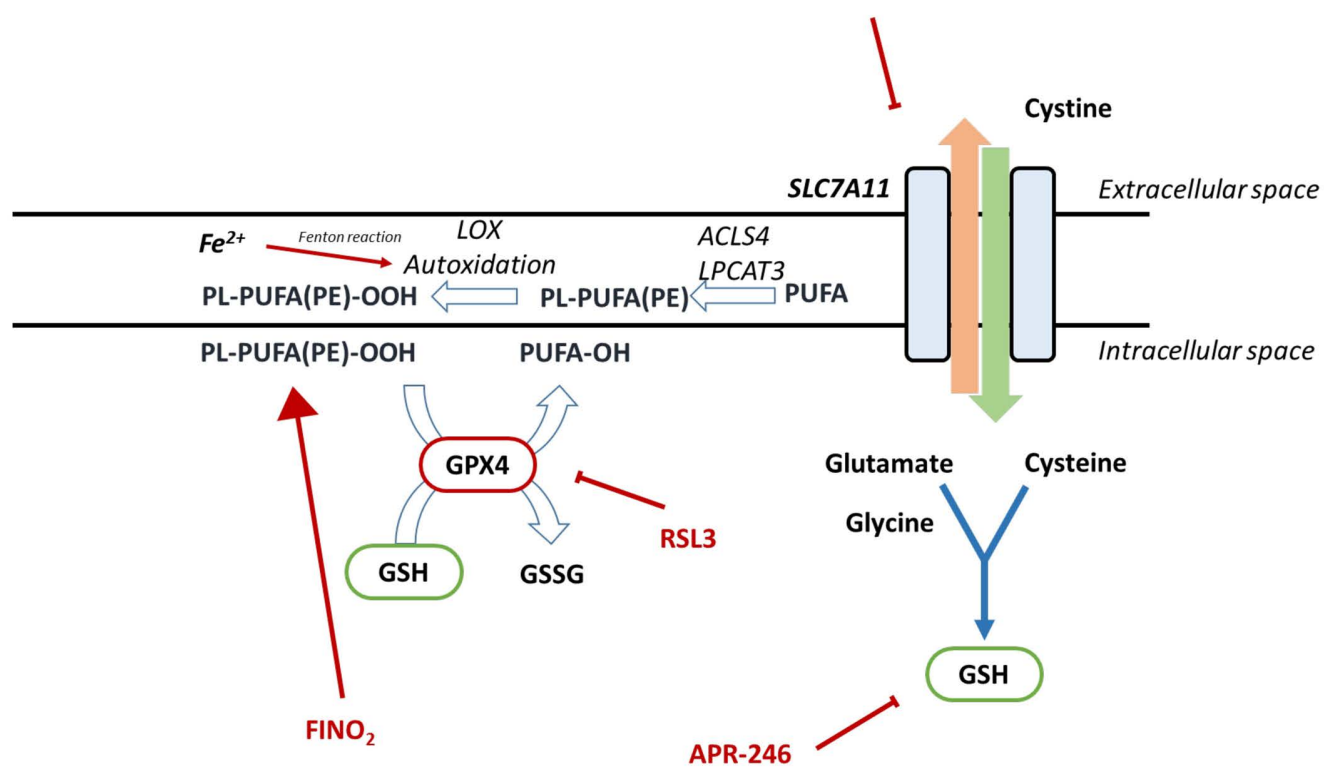

B

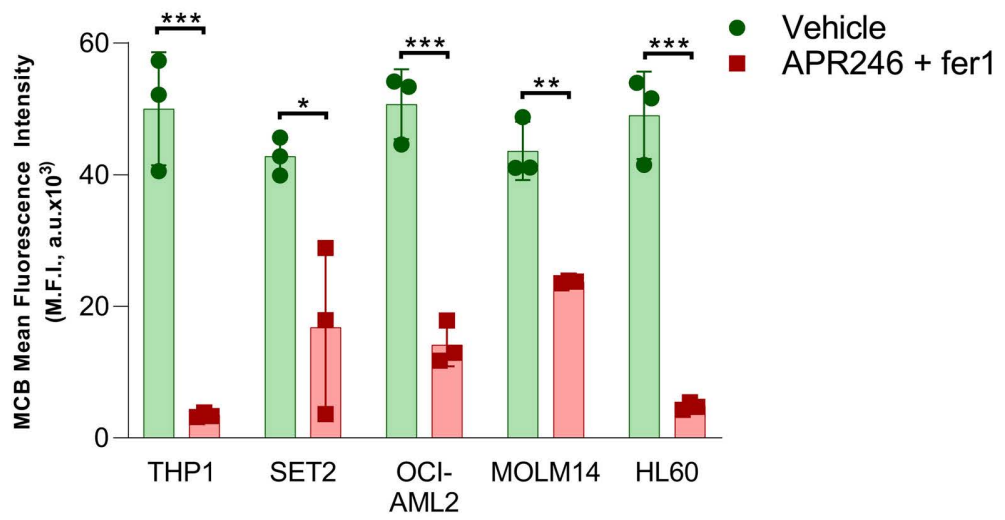

C

MOLM14

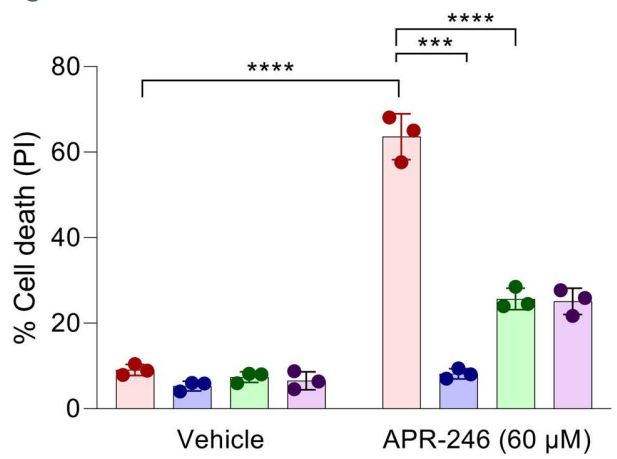

D

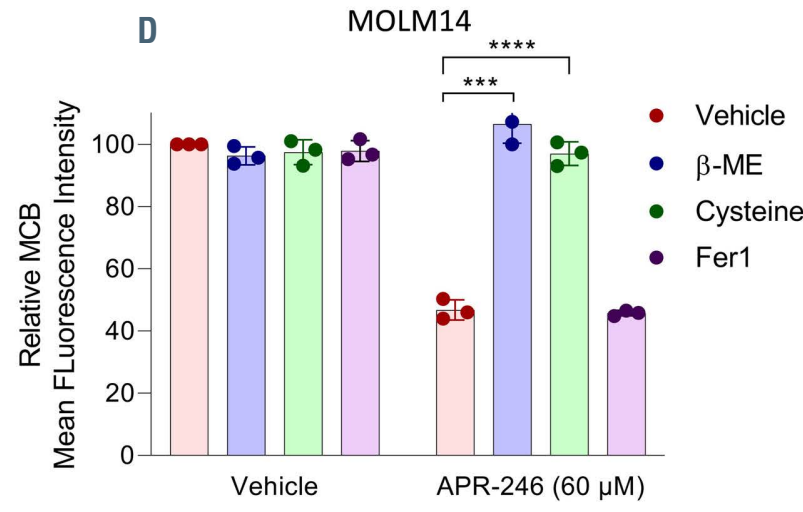

Figure 4. APR-246 induces glutathione depletion in acute myeloid leukemia cells. (A) Summary of the cellular pathways involved in ferroptosis. Ferroptosis execution is triggered by an iron-catalyzed excessive peroxidation of polyunsaturated fatty acids (PUFA)-containing phospholipids (PL-PUFA). Glutathione (GSH) and glutathione peroxidase 4 (GPX4) are the two key elements controlling the elimination of lipid peroxides. Solute carrier family 7 member 11 (SLC7A11) encodes the transporter subunit of the heterodimeric cystine-glutamate antiporter named system xc-. System xc- mediates cystine entry into the cell in exchange for glutamate. ${ }^{26}$ Once inside the cell, cystine is rapidly reduced to cysteine which is the limiting amino acid for GSH synthesis. SLC7A11 inhibition results in cellular cysteine depletion, which leads to the exhaustion of intracellular pool of GSH. GPX4 is a pleiotropic selenoprotein that uses GSH to selectively reduce lipid hydroperoxides to lipid alcohols, in order to protect the cells against membrane lipid peroxidation. ${ }^{29}$ GPX4 inhibition is either due to its direct inhibition or downregulation, or to GSH depletion via direct or indirect processes. The inhibition of GPX4 results in uncontrolled polyunsaturated fatty acid phospholipid (PL-PUFA) oxidation and fatty acid radical generation, leading to ferroptotic cell death. ACSL4: acyl-CoA synthetase long chain family member 4; LPCAT 3: lysophosphatidylcholine acyltransferase 3; ALOX: arachidonate lipoxygenase; PUFA: polyunsaturated fatty acid; PL: phospholipids; PE: phosphatidylethanolamine; GPX4: glutathione peroxidase 4. (B) GSH (mBCI) measurement in acute myeloid leukemia (AML) cell lines by flow cytometry (FCM) at 14 hours (h) post APR-246 and fer1 (10 $\mu$ M) treatment. APR-246 was used at $100 \mu \mathrm{M}$ for MOLM-14 and $50 \mu \mathrm{M}$ for other AML cell lines. Fer1 was associated to prevent cell death and allowed analysis of GSH depletion. Left panels show representative FCM quantification. $\mathrm{n}=3$. Error bars + standard deviation. Statistics by $t$-test. $* P<0.05, * * P<0.01, * * * P<0.0001$. (C and D) Cell death (\%) (C) and GSH (mBCl) measurement (D) for MOLM-14 at $24 \mathrm{~h}$ post-APR-246 treatment $(60 \mu \mathrm{M})$ with or without B-ME $(50 \mu \mathrm{M})$, cysteine $(50 \mu \mathrm{M})$ or Fer1 $(10 \mu \mathrm{M})$. Error bars + standard deviation. Statistics by $t$-test; $* P<0.05, * * P<0.01, * * * P<0.0001$. 


\section{Results}

\section{APR-246 induces ferroptosis in acute myeloid leukemia cells}

In order to determine the activity of APR-246 in AML, we assayed a set of 12 AML cell lines carrying diverse and representative molecular abnormalities, and five primary AML samples (Online Supplementary Table S1). Most of the AML cell lines and primary AML cells were sensitive to cell death induction by APR-246 (Figure 1A and B). The $\mathrm{IC}_{50}$ of APR-246 at $24 \mathrm{~h}$ for each cell line ranged from 11 $\mu \mathrm{M}$ to more than $200 \mu \mathrm{M}$, independently of their TP53 mutational status (Figure 1C). Previous in vivo human data have shown that the plasma concentrations of APR-246 in the $12 \mathrm{~h}$ following its intravenous administration range from 50 to $500 \mu \mathrm{M}$, suggesting that concentrations above $50 \mu \mathrm{M}$ are suitable for in vitro studies of the early effects of APR-246. ${ }^{19}$ In our subsequent experiments, we selected five AML cell lines sensitive to APR-246 in these concentration ranges, with or without TP53 mutations.

In order to investigate the mechanisms underlying APR-246 activity against AML cells, we exposed the cells treated with APR-246 to inhibitors of various cell death pathways (Figure 2A). The decrease in cell viability was almost completely rescued by either iron chelation via deferoxamine (DFO) or by the lipophilic antioxidant ferrostatin-1 (Fer1), demonstrating that cell death is both iron and reactive- oxygen species (ROS) dependent. Necrostatin-1 (Nec1) also consistently prevented cell death induced by APR-246 after short-term incubation in all AML cells lines. Nec1 has been used to define necroptotic cell death, but can also protect against ferroptosis through a target which is as yet unknown. ${ }^{20}$ Induction of necroptosis after treatment with APR-246 in our cells was excluded due to the absence of protection by more specific necroptosis inhibitors (Necrostatin 1s and Necrosulfonamide), ${ }^{21,22}$ and due to the absence of MLKL phosphorylation, a key marker in necroptosis ${ }^{21}$ (Online Supplementary Figure S1A and B). Inhibitors of autophagy (chloroquine) or apoptosis (OVD-OPH) failed to block APR-246-induced cell death. We confirmed that the mechanism of APR-246 induced cell death is distinct from apoptosis, as evidenced by the absence of caspases $3 / 8$ or PARP1 cleavage, including in TP53 mutated AML cell lines (Figure 2B; Online Supplementary Figure S2A to C). Notably, the protection against cell death observed with Fer1, DFO or Nec1 was partially lost at higher doses of APR-246 (Figure 2C) and at later time points (Figure 2D). However, apoptosis did not appear to be the mechanism of this late death, since caspases 3/8 and PARP1 were not cleaved and OVD-OPH was still unable to prevent the cell death (Online Supplementary Figure S2A to E). Examination of the ultrastructural changes induced by APR-246 treatment did not reveal any characteristic features of apoptosis (i.e., no plasma membrane blebbing, chromatin condensation or nuclear fragmentation) or autophagy (absence of autophagolysosomes). Necrotic cells were rare, and some mitochondria showed membrane rupture and reduced cristae (Figure 3A; Online Supplementary Figure S3). We analyzed the levels of endogenous lipid peroxidation - a hallmark of ferroptosis induction - following APR-246 treatment by flow cytometry analysis with C11-BODIPY staining. We observed a high induction of lipid ROS in AML cell lines and primary AML cells from two patients treated with APR-246 ex vivo. This increase in lipid ROS was fully reversed by Fer1, indicating that lipid peroxides had been newly generated (Figure $3 \mathrm{~B}$ and $\mathrm{C}$ ). All these results allowed to conclude that APR-246 induces early cell death by ferroptosis, a recently described non-apoptotic form of regulated cell death that links together membrane lipid peroxidation, cysteine and iron metabolism, glutathione peroxidase activity and oxidative stress (as summarized in Figure $4 \mathrm{~A}^{23-25}$ ). As previously reported, ${ }^{14,16}$ we observed that APR-246 treatment induced a dramatic decrease in GSH levels in AML cell lines (Figure 4B). Cysteine is the main biosynthetic precursor of GSH. Cysteine can be transported into cells via membrane transporters for neutral amino acids. However, in the extracellular space, cysteine is rapidly reduced to cystine. Thus the main source of intracellular cysteine comes from the entry of cystine into the cell via system xc- ${ }^{26} \beta$-mercaptoethanol ( $\beta$-ME) can promote cystine uptake through an alternative pathway. ${ }^{27}$ $\beta-M E$ was able to completely rescue the cell death and GSH depletion induced by APR-246 (Figure 4C and D). Cysteine treatment showed similar results as Fer1 treatment (Figure 4C and D). Altogether, these results demonstrate that APR-246 induces GSH depletion which induces ferroptosis in AML cells irrespective of their TP53 mutational status, and that ferroptosis induction is the main mechanism of cell death after early exposure to APR-246.

\section{Cystine uptake determined the sensitivity of acute myeloid leukemia cells to APR-246}

Since cysteine is a biosynthetic precursor of GSH, we asked whether the ability of cells to provide cysteine for GSH synthesis underlies the sensitivity to APR-246. Using FITC-labeled cystine, we showed that after exposure to APR-246, AML cells increased their uptake of cystine from the extracellular space (Figure 5A). Western blot analysis of the protein levels of SLC7A11 showed an increased amount of SLC7A11 (Figure 5B). This suggests that the cells enhanced cystine uptake by increasing SLC7A11 protein levels to maintain intracellular GSH levels after exposure to APR-246. We modulated the cystine uptake through SLC7A11 overexpression or inhibition. SLC7A11 overexpression in MOLM-14 and OCI-AML2 (Online Supplementary Figure S4) decreased the cell death and prevented the depletion of GSH induced by APR-246 (Figure $5 \mathrm{C}$ and $\mathrm{D}$ ). Then we showed that targeting the SLC7A11 cystine transporter by RNA interference reduced the basal uptake of cystine (Online Supplementary Figure $S 4 B$ and $C$ ) and had a very little effect on cell death (Online Supplementary Figure S4D), but strongly reduced cell proliferation in AML cells in vitro (Online Supplementary Figure S4E). Inhibition of SLC7A11 with RNA interference increased cell death and viability impairment, GSH depletion, and the accumulation of lipid peroxides induced by APR-246 (Figure 6A to C; Online Supplementary Figure S4F). Interestingly, basal GSH levels were not consistently affected by $\beta-M E$ addition, cysteine addition, or the overexpression or inhibition of SLC7A11, which suggests that the amount of GSH in basal conditions is not a reliable marker of cell cystine uptake ability (Figures 4D, 5D and 6C). Finally, targeting SLC7A11 with erastin, a potent inhibitor of system $\mathrm{xc}^{28}$ that showed variable sensitivity in our AML cell lines (Online Supplementary Figure S4G), had synergistic activity with APR-246 both on cell death and on cell viability 
impairment (Figure 6D and E; Online Supplementary Figure S5). The association of erastin and APR-246 also had a synergistic effect on cell viability in five primary AML samples (Figure 6F). There was no correlation between the basal levels of GPX4 and SLC7A11 proteins and the sensitivity to APR-246 (Online Supplementary Figure S6A and $B$ ). Altogether, these data suggest that the ability of AML cells to prevent lipid peroxides accumulation by increasing their cystine uptake to support GSH after exposure to APR-246 is a key determinant of the sensitivity to this compound.

The association of APR-246 and ferroptosis inducers has a synergistic anti-leukemic activity in vitro

We next determined whether targeting ferroptosis pathways in combination with APR-246 can increase the antileukemic activity of this compound, mimicking SLC7A11 inhibition. Downregulation of GPX4 by RNA interference
A

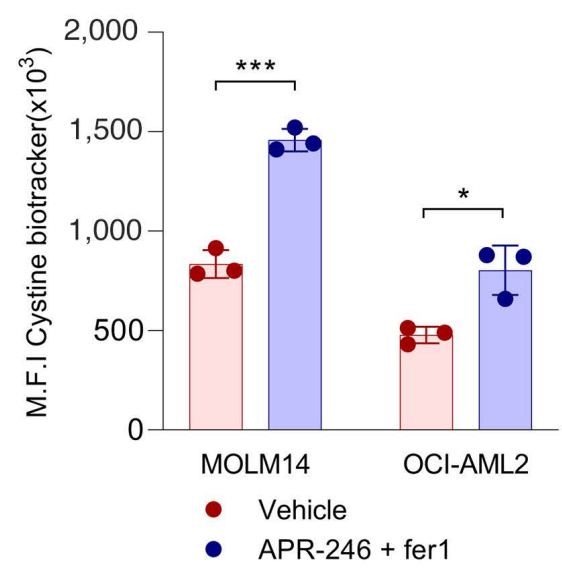

B

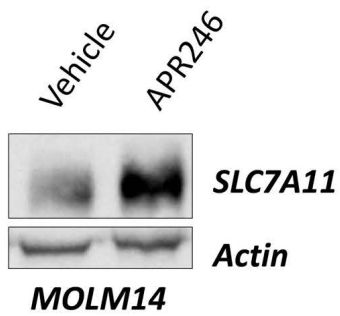

C

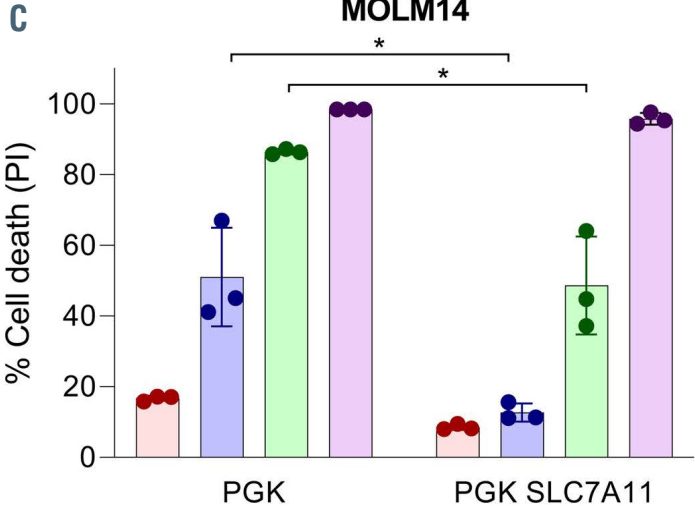

D

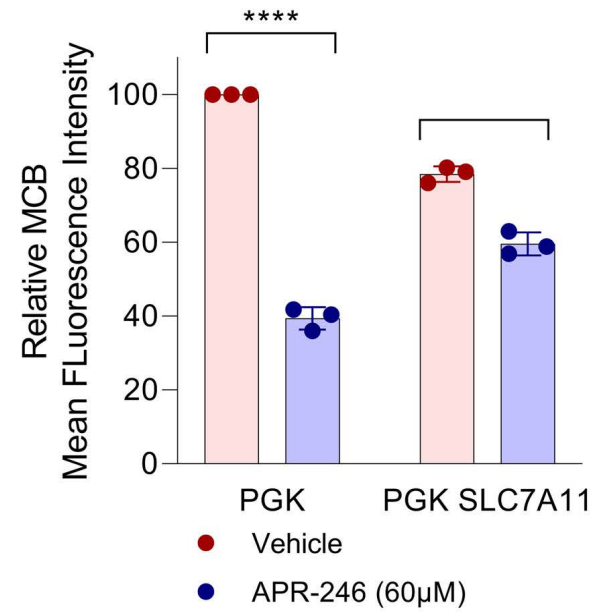

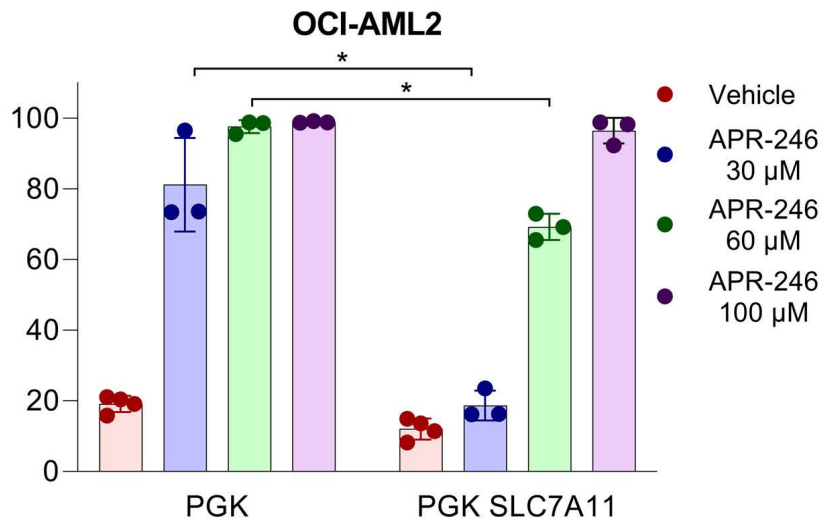

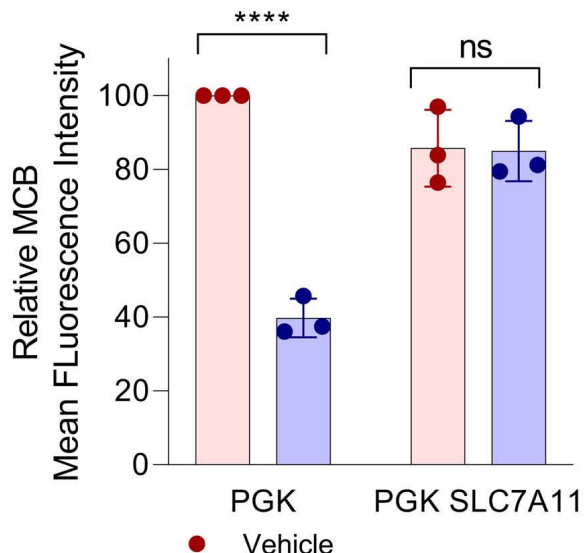

- $\mathrm{APR}-246(30 \mu \mathrm{M})$

Figure 5. SLC7A11 overexpression prevents glutathione depletion and cell death following APR-246 exposure. (A) Cystine uptake in MOLM-14 and OCI-AML2 cells lines at 16 hours $(\mathrm{h})$ post-APR-246 $(100 \mathrm{\mu M})$ and Fer1 $(10 \mathrm{\mu M})$ treatment. Fer1 was associated to prevent cell death and allowed analysis of cystine uptake. (B) Immunoblotting analysis of SLC7A11 in MOLM-14 cells treated for $16 \mathrm{~h}$ with dimethyl sulfoxide (DMSO) or APR-246 ( $\mathrm{n}=2$ ). $\beta$-actin was used as a loading control. (C and $\mathrm{D})$ Cell death $(\%)(\mathrm{C})$ and glutathione $(\mathrm{GSH})(\mathrm{mBCl})$ measurement $(\mathrm{D})$ of the indicated cells at $20 \mathrm{~h}$ post-APR-246 treatment $(\mathrm{n}=3)$. For GSH measurement, Fer1 was associated to prevent cell death and allowed analysis of GSH depletion. Error bars \pm standard deviation. Statistics by $t$-test. $* P<0.05, * * P<0.01, * * \star P<0.0001$. 
A

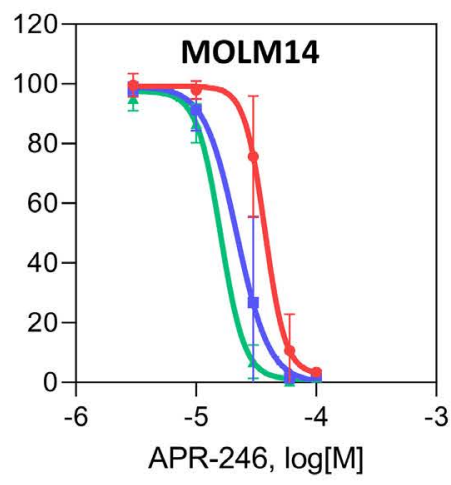

MOLM14

B

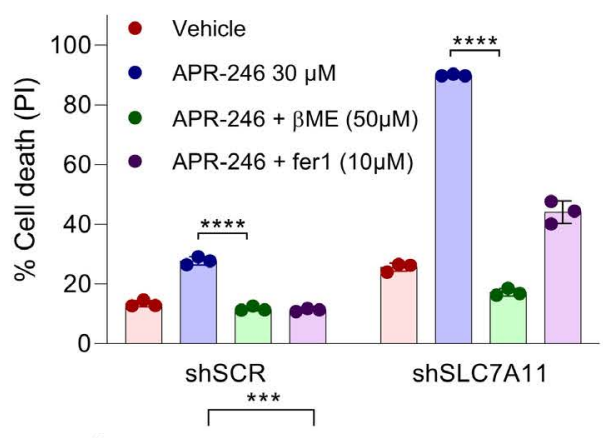

C

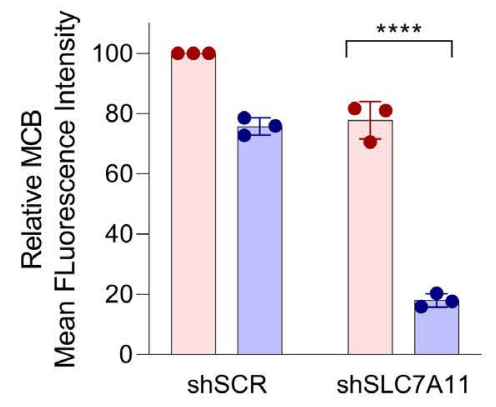

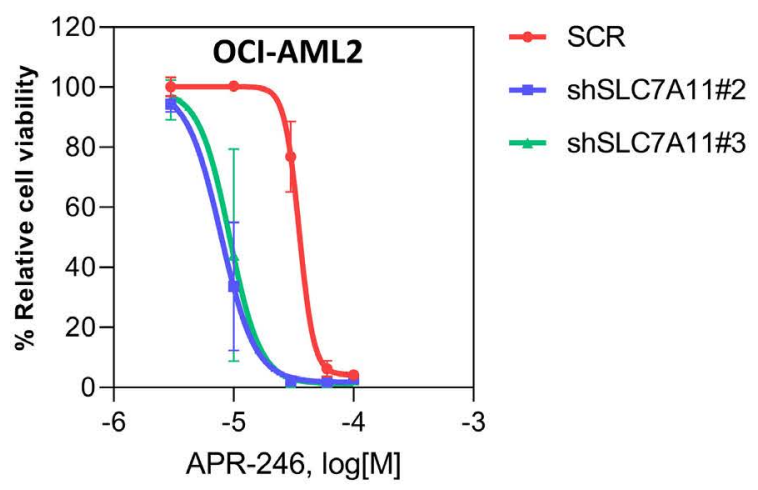

OCI-AML2
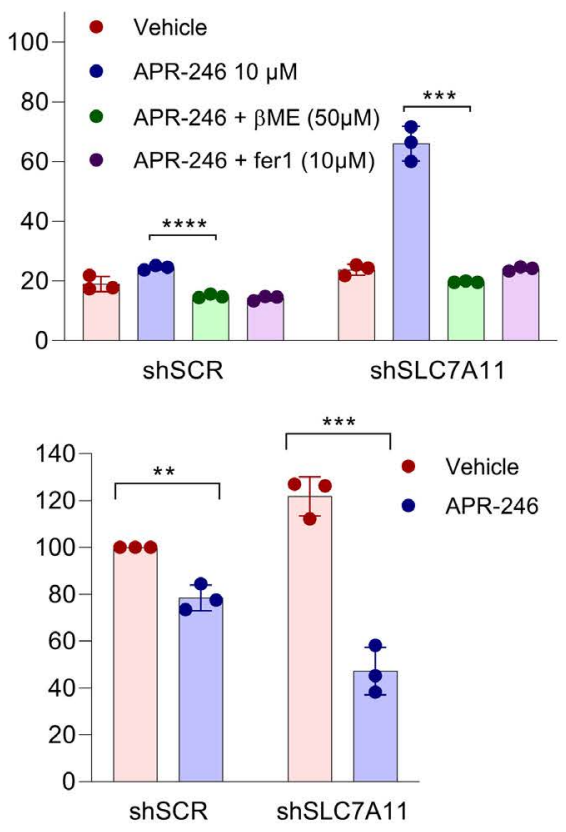

D

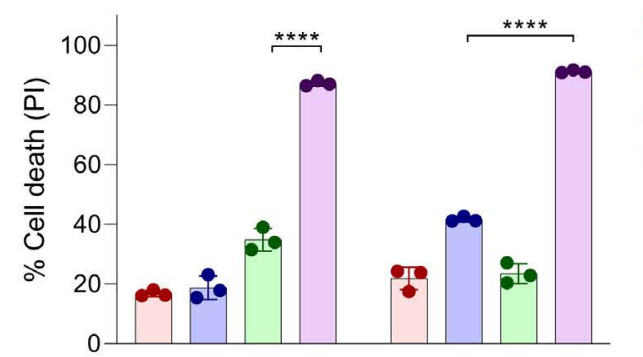

MOLM14

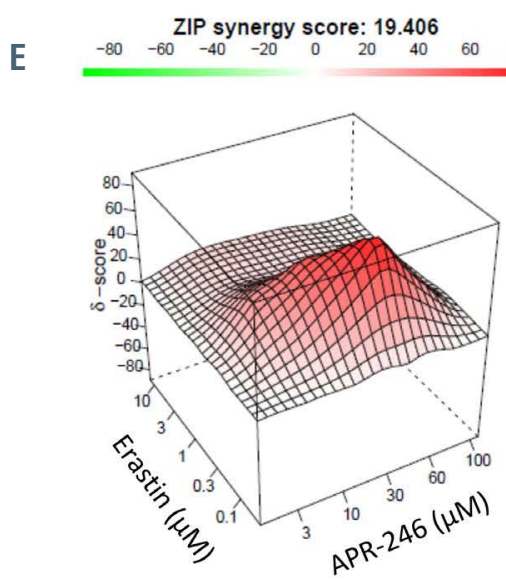

OCI-AML2
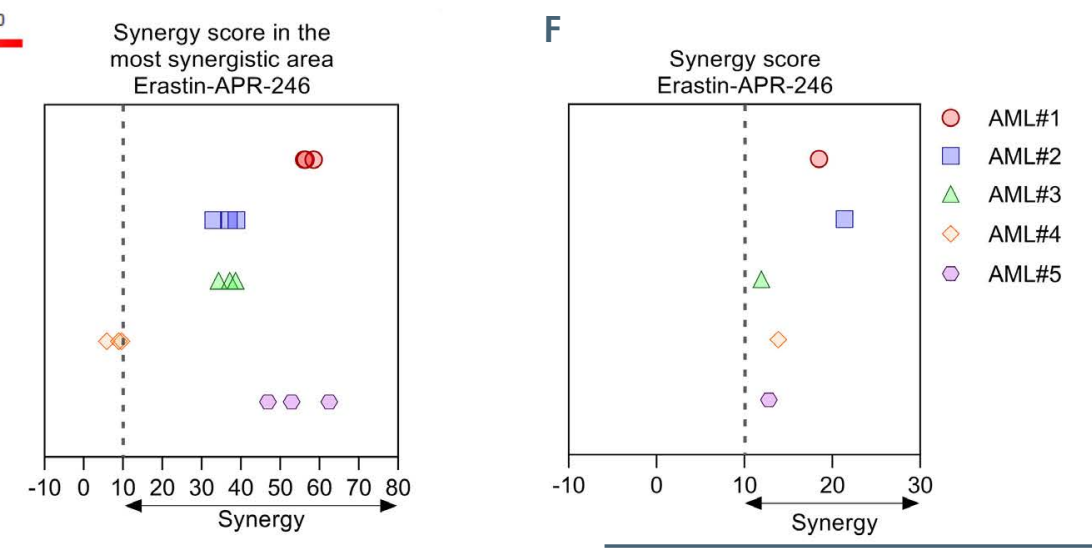

Figure 6. Legend on following page. 
Figure 6. SLC7A11 inhibition sensitizes cells to APR-246. (A) Viability curves for MOLM-14 and OCl-AML2 with small hairpin RNA control (shSCR) or doxycyclineinducible shRNA (shSLC7A11) cells at 16 hours $(\mathrm{h})$ post APR-246 treatment. Prior to adding APR-246, the cells were treated for 3 days with doxycycline ( $\mathrm{n}=3$ ). Error bars \pm standard deviation (SD). (B and C) Cell death (\%) (B) and glutathione (GSH) (mBCl) measurement (C) of the indicated cells at $20 \mathrm{~h}$ post-APR-246 (MOLM-14 $30 \mu \mathrm{M}, \mathrm{OCl}-\mathrm{AML} 210 \mu \mathrm{M})$ treatment $(\mathrm{n}=3)$. For GSH measurement, Fer1 was associated to prevent cell death and allowed analysis of GSH depletion. Prior to adding APR-246, the cells were treated for 3 days with doxycycline $(n=3)$. Error bars \pm SD. Statistics by $t$-test. $* P<0.05, * * P<0.01$, $* * * P<0.0001$. (D) Cell death (\%) for the indicated cell types at $24 \mathrm{~h}$ post APR-246 (MOLM-14 $30 \mu \mathrm{M}$, OCl-AML2 $10 \mu \mathrm{M}$ ) and or erastin (MOLM-14 $100 \mathrm{nM}$, OCl-AML2 $1 \mu \mathrm{M})$. Error bars \pm SD. Statistics by t-test: $* P<0.05, * * P<0.01$. (E) Illustrative synergy map (left panel) of $24 \mathrm{~h}$ co-treatment of MOLM-14 cells with APR-246 and erastin. The mean cell viability of three independent experiments was used. Mean synergy scores of the most synergistic area of $24 \mathrm{~h}$ co-treatment of AML cell lines with APR-246 and erastin ( $\mathrm{n}=3$ ). ( $\mathrm{F}$ ) Mean synergy score of the $48 \mathrm{~h}$ co-treatment of primary AML cells with APR-246 and erastin $(n=1)$.

resulted in cell death (Online Supplementary Figure S7A and $B)$. Our AML cell lines panel showed variable sensitivity to two ferroptosis-inducing drugs: RSL3, a direct GPX4 inhibitor; ${ }^{29}$ and FINO2, an indirect GPX4 inhibitor and direct iron oxidant ${ }^{30}$ (Online Supplementary Figure S8). We observed that knockdown of GPX4 increased the impairment of cell viability induced by APR-246 (Figure 7A). RSL3 and FINO2 in association with APR-246 synergistically decreased cell viability in AML cell lines (Figure 7B and C; Online Supplementary Figures S9 and 10). Collectively, these results show that pharmacological or genetic activation of ferroptosis enhances the antileukemic activity of APR-246 in AML.

\section{Genetic invalidation of SLC7A11 has synergistic anti-leukemic activity with APR-246 in vivo}

We then examined whether inhibition of GSH synthesis through SLC7A11 inhibition could interfere with AML persistence and could enhance APR-246 activity in vivo. We engrafted MOLM14 cells transduced with either control (shSCR) or anti-SLC7A11 (shSLC7A11) doxycyclineinducible shRNA (Figure 8A). After induction of shRNA expression in vivo, we treated the mice with a 4-day APR246 regimen in order to mimic the therapeutic schedule used in clinical trials of APR-246. ${ }^{16}$ This treatment scheme varied from those previously published in mice in terms of treatment duration. Indeed, in these studies, APR-246 was administrated for 7 to 28 days, and reduction of tumor volume after 4 days of treatment was minimal at best. ${ }^{7,8,14,16}$ However, our aim was not to assess the efficacy of APR-246 alone but to demonstrate that its association with SLC7A11 inhibition enhanced its anti-leukemic activity. As expected, APR-246 alone did not reduce BM tumor cell burden. SLC7A11 knockdown significantly reduced tumor cell burden in the BM (Figure $8 \mathrm{~B}$ and $\mathrm{C}$ ). Moreover, the decrease in BM tumor cell burden was enhanced when APR-246 treatment was combined to SLC7A11 knockdown (Figure 8B and C). Overall, these results showed that inhibition of anti-ferroptosis mechanisms enhanced the anti-leukemic activity of APR-246 in vivo.

\section{Discussion}

APR-246 can restore the wild-type conformation of mutant p53 protein, therefore inducing apoptosis and inhibition of tumor growth in mice. ${ }^{6}$ Thus, APR-246 is one of the most promising compounds in clinical development for TP53 mutated cancers. Controversies exist over the TP53 mutation status dependencies of APR-246. ${ }^{31}$ Some studies reported that APR-246 acts independently of its ability to reactivate mutant p53 protein. ${ }^{12,32-37}$ Tessoulin et al. demonstrated that myeloma cells are highly sensitive to APR-246, independently of their TP53 status. ${ }^{14}$ In this cancer, APR-246 induces cell death by impairing GSH/ROS balance and acts synergistically with L-buthionine sulphoximine to inhibit myeloma growth in vivo. ${ }^{14}$ In ARID1A-deficient cancers, GSH was the major target of APR-246 and was the basis of the high sensitivity of these cancer cells to this coumpound. ${ }^{38}$ In esophageal cancer, Liu et al. showed that mutants p53 bind to the antioxidant transcription factor NRF2, leading to a decreased expression of SLC7A11 which sensitizes cells to GSH depletion by APR-246. ${ }^{16}$

Paradoxically, while APR-246 clinical development is the most advanced in AML with TP53 mutation, the effects of APR-246 have been little studied in this disease. Two studies showed that APR-246 induced in vitro cell death in a large number of leukemic cells from patients, alone or in association with chemotherapies. ${ }^{39,40}$ In both studies, the cytotoxicity of APR-246 was independent of the TP53 mutational status. The mechanisms of action of APR-246 was investigated in AML cell lines with TP53 mutations, and more specifically studied its association with 5-azacytidine which is currently used in clinical trials. ${ }^{8}$ It was shown that in TP53-mutated myelodysplastic syndromes (MDS) and AML, APR-246 can reactivate the p53 pathway and induce an apoptotic transcriptional program, with synergistic effects of APR-246 and azacytidine.

In this context, our study strongly showed that APR-246 induced cell death in AML cells irrespective of their TP53 mutational status. APR-246 depleted intracellular GSH and induced lipid peroxide production, which led to ferroptosis induction. The ability of AML cells to detoxify lipid peroxides primed their sensitivity to APR-246 treatment. Additionally, we uncovered that inhibition of antiferroptosis mechanisms enhanced the anti-leukemic activity of APR-246 both in vitro and in vivo. We confirm the TP53 independence and GSH depletion and we demonstrated that APR-246 induces ferroptosis. ${ }^{14,16}$ Ferroptosis is rapidly induced after GPX4 inactivation and cell death occurs in the first 24 hours post-treatment or administration. ${ }^{23,29}$ The observation that protection against cell death by ferroptosis inhibitors decreases after 24 hours of exposure suggests that other cell death mechanisms might be involved after this early phase and that they may have masked the earlier induction of ferroptosis. The effect of APR-246 might be also different in AML cells in comparison to solid cancers.

Our study might have several important implications for the management of MDS and AML patients. First, since APR-246 acts independently of TP53 mutational status, this treatment could be used in a broader panel of AML patients. Future study will need to identify predictive elements of the sensitivity of AML to APR-246 and the induction of ferroptosis. The mechanism of action of APR-246, which is based on GSH depletion and induction of ferroptosis, makes it the first ferroptosis-inducing agent currently used therapeutically in humans. Using ferropto- 
A
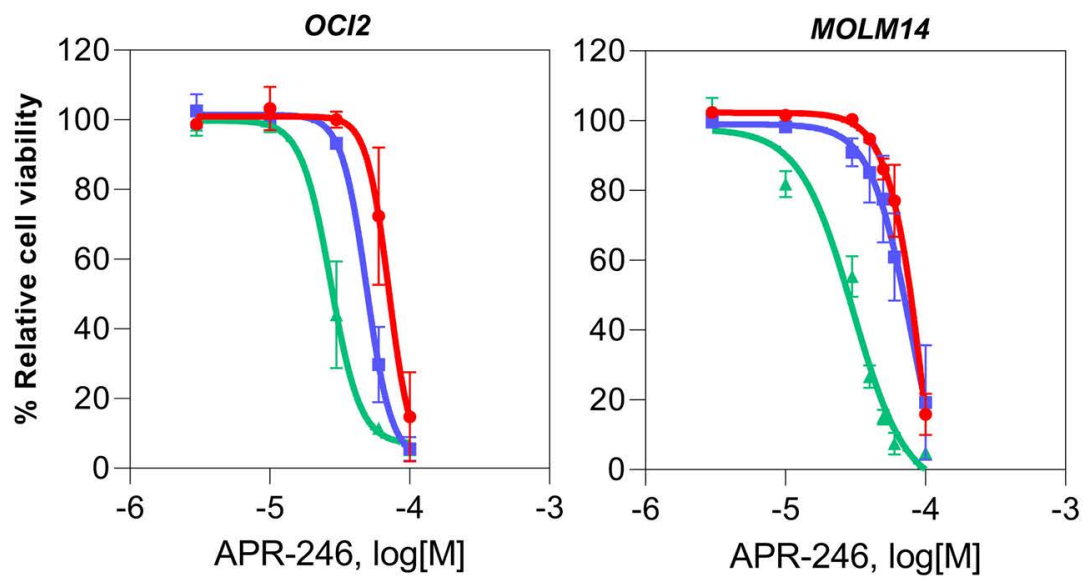

$\begin{array}{lcc}\text { IC }_{50} \text { APR-246 } & \text { OCI-AML2 } & \text { MOLM14 } \\ \text { - shSCR } & 70 \mu \mathrm{M} & 105 \mu \mathrm{M} \\ =\text { shGPX4\#1 } & 49 \mu \mathrm{M} & 79,7 \mu \mathrm{M} \\ - \text { shGPX4\#2 } & 27 \mu \mathrm{M} & 30,3 \mu \mathrm{M}\end{array}$

B
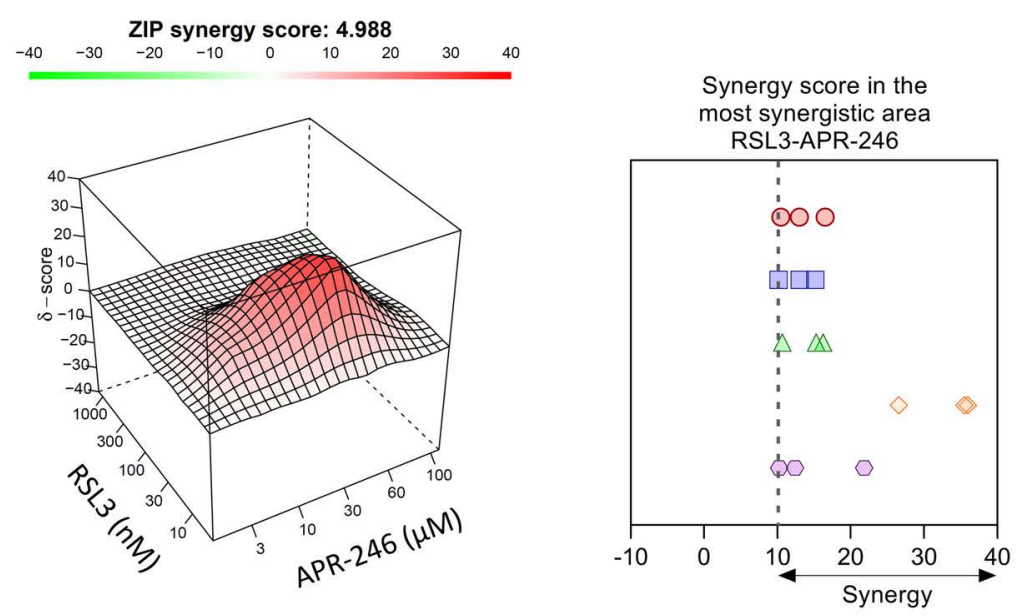

C
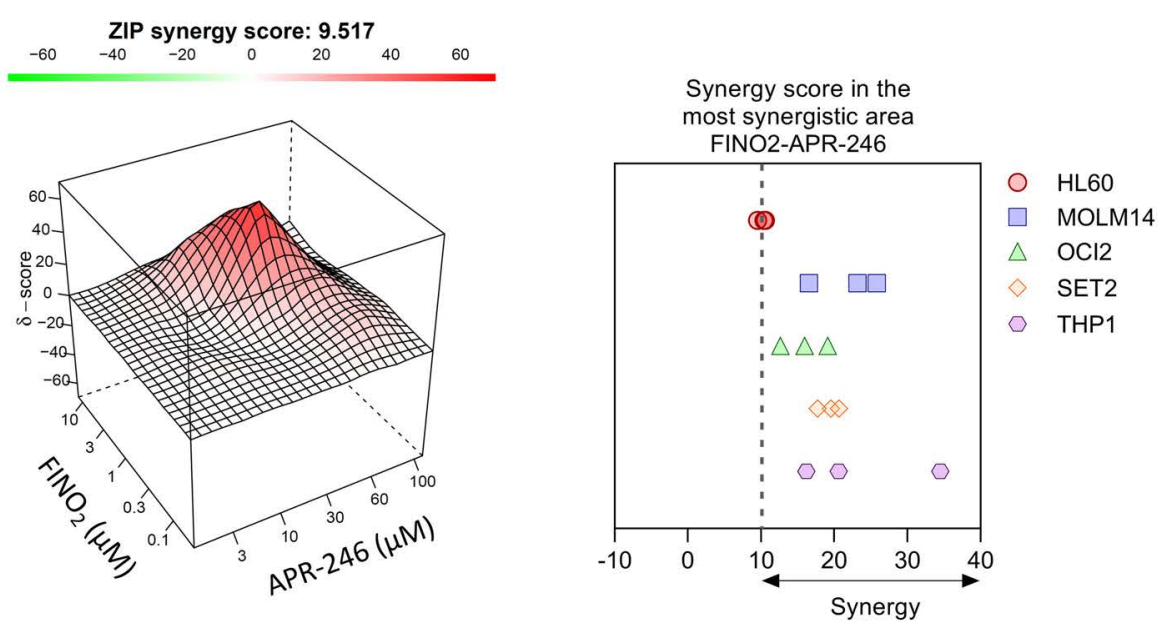

Figure 7. The combination of APR-246 with ferroptosis inducers has synergistic anti-leukemic effects in acute myeloid leukemia in vitro. (A) Viability curves for MOLM-14 and OCI-AML2 cells with or without GPX4 inducible small hairpin RNA (shRNA) at 16 hours (h) post APR-246 treatment. Prior to adding APR-246, the cells were treated for 2 days with doxycycline $(n=3)$. Error bars \pm standard deviation. (B) Illustrative synergy map (left panel) of $24 \mathrm{~h}$ co-treatment of MOLM-14 cells with APR-246 and RSL3. The mean cell viability of three independent experiments was used. Mean synergy scores of the most synergistic area of $24 \mathrm{~h}$ co-treatment of acute myeloid leukemia (AML) cell lines with APR-246 and RSL3 ( $n=3$ ).(C) Illustrative synergy map (left panel) of 24 h co-treatment of MOLM-14 cells with APR-246 and FINO2. The mean cell viability of three independent experiments was used. Mean synergy scores of the most synergistic area of $24 \mathrm{~h}$ co-treatment of AML cell lines with APR-246 and FINO2 ( $n=3)$. 
A

IV injection of MOLM14 ShSCR or VOLM14 ShSLC7A11

cells in NSG mice

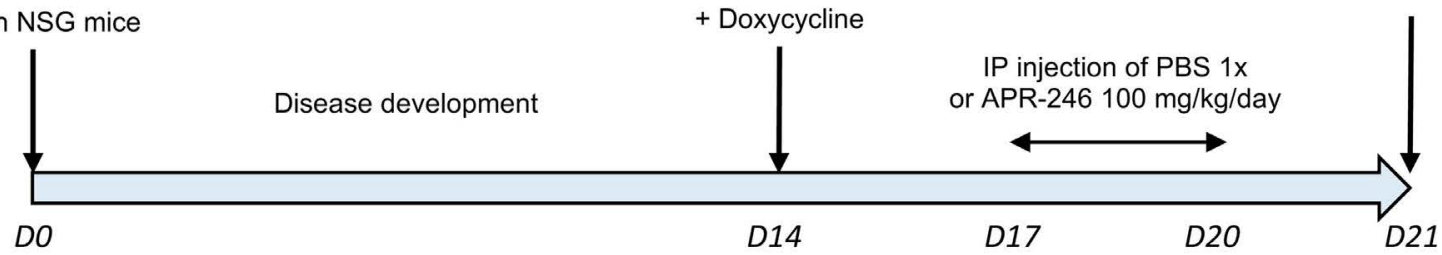

B
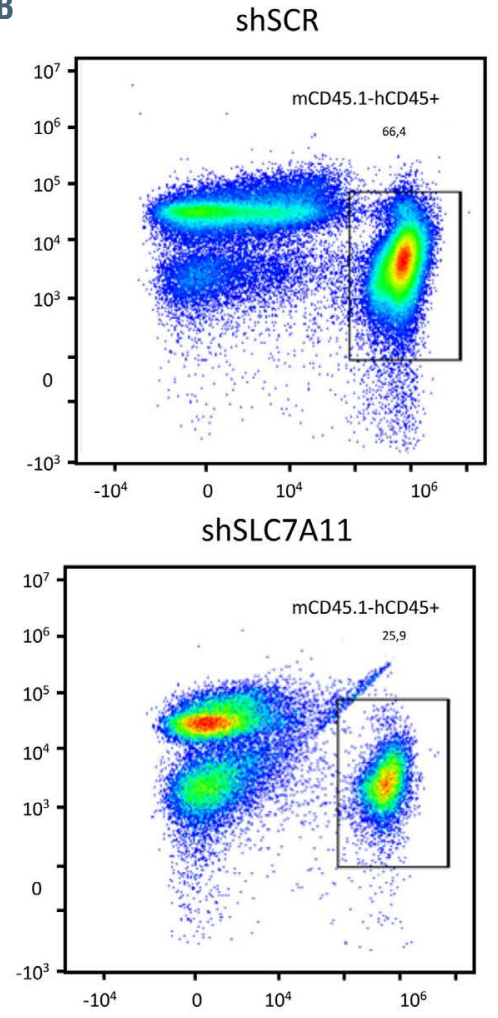

ShSCR + APR-246
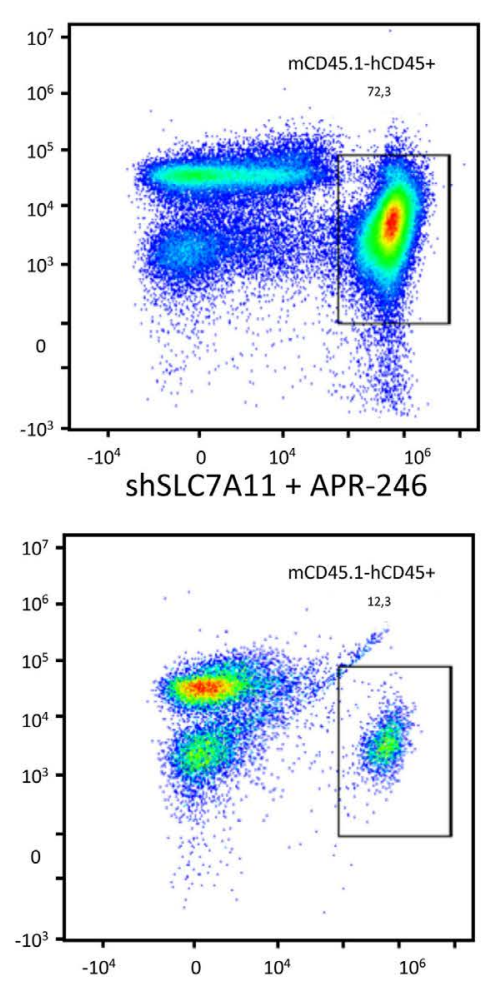

Group 1 ( $n=7)$ : shSCR + Vehicle Group 2 ( $n=8)$ : shSLC7A $11+$ Vehicle Group $3(n=7)$ : shSCR + APR-246 Group $4(n=8)$ : shSLC7A11 + APR-246

C Bone marrow and spleen

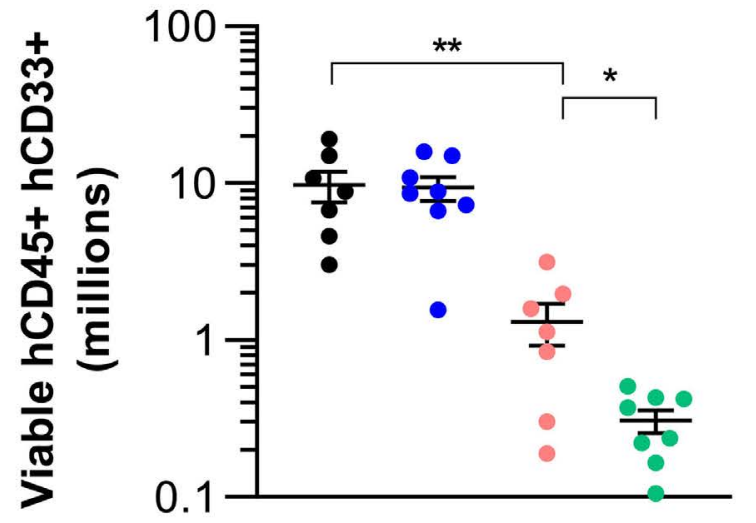

- $\quad$ ShSCR

- $\quad$ shSCR + APR-246

- $\quad$ shSLC7A11

- $\quad$ shSLC7A11 + APR-246

Figure 8. The combination of APR-246 with SLC7A11 inhibition has synergistic anti-leukemic effects in acute myeloid leukemia in vivo. (A) Design of the in vivo experiment. (B) Representative flow cytometry analysis of bone marrow cells marked with mCD45.1 and hCD45 from each treatment subgroup. (C) Viable mCD45.1$\mathrm{hCD} 45^{+} \mathrm{hCD} 33^{+}$cell counts in the bone marrow of the different treatment subgroups. Bars represent mean of all experiments and errors denote \pm standard deviations. Statistics by $t$-test; $* P<0.05, * * P<0.01, * * * P<0.0001$. 
sis induction to treat cancer is an emerging field in oncology research. Renal cancer cells have been reported as highly dependent on the GSH pathway for ROS detoxification, including lipid peroxides, and targeting components of this pathway is an effective strategy for the treatment of this disease. ${ }^{41}$ Other studies have elegantly highlighted the higher sensitivity to ferroptosis of cancer cells that are resistant to conventional therapy. ${ }^{42,43}$ In AML, data about ferroptosis are scarce. An in vitro study showed that the ferroptosis inducer erastin enhances sensitivity of AML cells to chemotherapeutic agents. ${ }^{44}$ Jones et al. recently reported that cysteine depletion leads to GSH exhaustion and ROS-low leukemic stem cell eradication in AML. ${ }^{45}$ Thus, APR-246 could act on these cell pools that are poorly sensitive to conventional therapy, and which are at the origin of frequent therapeutic failures in AML.

From a clinical perspective, this mechanism of action might be relevant. Indeed, iron chelators are frequently used for the treatment of iron overload due to red blood cell transfusions and dyserythropoïesis in MDS/AML patients. Several studies have reported beneficial effects of iron chelation therapy on overall survival in MDS patients with iron overload. ${ }^{46}$ However, iron chelators are recognized as canonical ferroptosis inhibitors. Therefore, caution should be exercised regarding co-administration of iron chelators which may antagonize the anti-leukemic activity of APR-246, as we observed in vitro. Moreover, the phase II studies of APR-246 in MDS/AML reported the occurrence of neurological adverse events in over a third of patients treated with APR-246. ${ }^{10,11}$ Recently, ferroptosis has been implicated in the pathogenesis of several neurological disorders, especially neurodegenerative disorders. ${ }^{47,48}$ One hypothesis could be that the neurological side effects observed after administration of APR-246 are linked to its ability to deplete GSH in neuronal cells. Consequently, anti-ferroptosis agents, such as iron chelators or vitamin E, could be valuable drugs to treat these side effects. Finally, our study highlights that ferroptosis induction may represent a new target in $\mathrm{AML}$, opening new therapeutic strategies based on disease-specific vulnerabilities. The effect of ferroptosis induction-based treatments on normal hematopoietic cells and their value compared to standard-of-care AML therapies will be important to evaluate in the future.

\section{Disclosures}

No conflicts of interest to disclose.

\section{Contributions}

JD, LCA, EG, TH performed experiments; CL, JES, JT, NG and $M G$ performed in vivo experiments; NC, $O K$ and $M F$ provided AML samples; NC, PM, JES, NJ and JT analyzed the results and corrected the manuscript; $R B$ performed experiments, analyzed data, and wrote the manuscript; $D B$ designed and supervised the research program, analyzed data, and wrote the manuscript. All authors approved the final version of the manuscript.

\section{Acknowledgments}

We thank Alain Schmitt and the cell imagery department at the Cochin Institute for performing the transmission electron microscopy. We thank Tata Jojo for manuscript proofreading. We also thank the CYBIO cytometry-department at the Cochin Institute. We further thank Aprea Therapeutics for providing the $A P R-246$ used in the in vivo study.

\section{Funding}

This work was supported by grants from the Association de Recherche Contre le Cancer (ARC; aides doctorales $R B$, grant $n^{\circ}$ DOC20170505807 and DOC20190508975; aides jeune chercheur TH, grant $\left.n^{\circ} M 2 R 20180507379\right)$, from the Institut National du Cancer (JD, grant $\left.n^{\circ} A S C 16046 K S A\right)$, from the Ligue Nationale Contre le Cancer (LNCC; DB, Equipe Labellisée EL2017. N $N^{\circ}$ Projet: ELFUZ17337; NG, grant $n^{\circ}$ IP/SCG/JD-16129) and from association Laurette Fugain (grant $\left.n^{\circ} A L F 2018 / 02\right)$.

\section{References}

1.Döhner H, Estey E, Grimwade D, et al. Diagnosis and management of AML in adults: 2017 ELN recommendations from an international expert panel. Blood. 2017;129(4):424-447.

2. Stone RM, Mandrekar SJ, Sanford BL, et al. Midostaurin plus chemotherapy for acute myeloid leukemia with a FLT3 Mutation. N Engl J Med. 2017;377(5):454-464

3. Stein EM, DiNardo CD, Pollyea DA, et al. Enasidenib in mutant-IDH2 relapsed or refractory acute myeloid leukemia. Blood. 2017;130(6):722-731.

4. DiNardo CD, Stein EM, de Botton S, et al. Durable remissions with Ivosidenib in IDH1-mutated relapsed or refractory AML. N Engl J Med. 2018;378(25):2386-2398.

5. DiNardo CD, Pratz K, Pullarkat V, et al. Venetoclax combined with decitabine or azacitidine in treatment-naive, elderly patients with acute myeloid leukemia. Blood. 2019;133(1):7-17.

6. Bykov VJN, Issaeva N, Shilov A, et al. Restoration of the tumor suppressor function to mutant p53 by a low-molecular-weight compound. Nat Med. 2002;8(3):282-288.

7. Saha MN, Jiang H, Yang Y, Reece D, Chang
H. PRIMA-1Met/APR-246 displays high antitumor activity in multiple myeloma by induction of p73 and Noxa. Mol Cancer Ther. 2013;12(11):2331-2341.

8. Maslah N, Salomao N, Drevon L, et al. Synergistic effects of PRIMA-1Met (APR246) and Azacitidine in TP53-mutated myelodysplastic syndromes and acute myeloid leukemia. Haematologica. 2020; 105(6):1539-1551.

9. Nahi H, Merup M, Lehmann S, et al. PRIMA-1 induces apoptosis in acute myeloid leukaemia cells with p53 gene deletion. Br J Haematol. 2006;132(2):230236.

10. Sallman DA, DeZern AE, Garcia-Manero $G$, et al. Phase 2 results of APR-246 and Azacitidine (AZA) in patients with TP53 mutant myelodysplastic syndromes (MDS) and oligoblastic acute myeloid leukemia (AML). Blood. 2019;134(Suppl 1):S676.

11. Cluzeau T, Sebert M, Rahmé R, et al. APR246 combined with Azacitidine (AZA) in TP53 mutated myelodysplastic syndrome (MDS) and acute myeloid leukemia (AML). A phase 2 study by the Groupe Francophone Des Myélodysplasies (GFM). Blood. 2019;134(Suppl 1):S677.

12. Lambert JMR, Gorzov P, Veprintsev DB, et al. PRIMA-1 reactivates mutant p53 by covalent binding to the core domain Cancer Cell. 2009;15(5):376-388.

13. Zhang Q, Bykov VJN, Wiman KG, Zawacka-Pankau J. APR-246 reactivates mutant p53 by targeting cysteines 124 and 277. Cell Death Dis. 2018;9(5):1-12.

14. Tessoulin B, Descamps G, Moreau P, et al. PRIMA-1Met induces myeloma cell death independent of p53 by impairing the GSH/ROS balance. Blood. 2014; 124(10):1626-1636.

15. Bykov VJN, Zhang Q, Zhang M, Ceder S, Abrahmsen L, Wiman KG. Targeting of mutant p53 and the cellular redox balance by APR-246 as a strategy for efficient cancer therapy. Front Oncol. 2016;6:21.

16. Liu DS, Duong CP, Haupt S, et al. Inhibiting the system $\mathrm{xC}$-/glutathione axis selectively targets cancers with mutant-p53 accumulation. Nat Commun. 2017:8:14844.

17. Jacque N, Ronchetti AM, Larrue C, et al. Targeting glutaminolysis has antileukemic activity in acute myeloid leukemia and synergizes with BCL-2 inhibition. Blood 2015;126(11):1346-1356.

18. Ianevski A, He L, Aittokallio T, Tang J. SynergyFinder: a web application for analyzing drug combination dose-response matrix data. Bioinformatics. 2017;33(15): 2413-2415. 
19. Lehmann S, Bykov VJN, Ali D, et al. Targeting p53 in vivo: a first-in-human study with p53-targeting compound APR246 in refractory hematologic malignancies and prostate cancer. J Clin Oncol. 2012;30(29):3633-3639.

20. Friedmann Angeli JP, Schneider M, Proneth $\mathrm{B}$, et al. Inactivation of the ferroptosis regulator Gpx4 triggers acute renal failure in mice. Nat Cell Biol. 2014;16(12):1180-1191.

21. Sun L, Wang H, Wang Z, et al. Mixed lineage kinase domain-like protein mediates necrosis signaling downstream of RIP3 kinase. Cell. 2012;148(1-2):213-227.

22. Takahashi N, Duprez L, Grootjans S, et al. Necrostatin-1 analogues: critical issues on the specificity, activity and in vivo use in experimental disease models. Cell Death Dis. 2012;3(11):e437.

23. Dixon SJ, Lemberg KM, Lamprecht MR, et al. Ferroptosis: an iron-dependent form of nonapoptotic cell death. Cell. 2012; 149(5):1060-1072.

24. Stockwell BR, Friedmann Angeli JP, Bayir $\mathrm{H}$, et al. Ferroptosis: a regulated cell death Nexus linking metabolism, redox biology, and disease. Cell. 2017;171(2):273-285.

25. Feng H, Stockwell BR. Unsolved mysteries: How does lipid peroxidation cause ferroptosis? PLoS Biol. 2018;16(5):e2006203.

26. Koppula P, Zhang Y, Zhuang L, Gan B. Amino acid transporter SLC7A11/xCT at the crossroads of regulating redox homeostasis and nutrient dependency of cancer. Cancer Commun (Lond). 2018;38(1):12.

27. Ishii T, Bannai S, Sugita Y. Mechanism of growth stimulation of L1210 cells by 2 mercaptoethanol in vitro. Role of the mixed disulfide of 2-mercaptoethanol and cysteine. J Biol Chem. 1981;256(23):1238712392

28. Dixon SJ, Patel DN, Welsch $M$, et al. Pharmacological inhibition of cystine-glutamate exchange induces endoplasmic reticulum stress and ferroptosis. Elife. 2014;3:e02523.

29. Yang WS, SriRamaratnam R, Welsch ME, et al. Regulation of ferroptotic cancer cell death by GPX4 Cell. 2014:156(1):317-331.

30. Gaschler MM, Andia AA, Liu $\mathrm{H}$, et al. FINO2 initiates ferroptosis through GPX4 inactivation and iron oxidation. Nat Chem Biol. 2018;14(5):507-515.

31. Perdrix A, Najem A, Saussez $S$, et al PRIMA-1 and PRIMA-1Met (APR-246): from mutant/wild type p53 reactivation to unexpected mechanisms underlying their potent anti-tumor effect in combinatorial therapies. Cancers (Basel). 2017;9(12):172.

32. Bykov VJN, Zache N, Stridh H, et al. PRIMA-1(MET) synergizes with cisplatin to induce tumor cell apoptosis. Oncogene. 2005;24(21):3484-3491.

33. Lambert JMR, Moshfegh A, Hainaut P, Wiman KG, Bykov VJN. Mutant p53 reactivation by PRIMA-1 MET induces multiple signaling pathways converging on apoptosis. Oncogene. 2010;29(9):1329-1338.

34. Duan W, Gao L, Wu X, et al. MicroRNA34a is an important component of PRIMA1-induced apoptotic network in human lung cancer cells. Int J Cancer. 2010;127 (2):313-320.

35. Roh J-L, Kang SK, Minn I, Califano JA, Sidransky D, Koch WM. p53-Reactivating small molecules induce apoptosis and enhance chemotherapeutic cytotoxicity in head and neck squamous cell carcinoma. Oral Oncol. 2011:47(1):8-15

36. Izetti $\mathrm{P}$, Hautefeuille A, Abujamra AL, et al. PRIMA-1, a mutant p53 reactivator, induces apoptosis and enhances chemotherapeutic cytotoxicity in pancreatic cancer cell lines. Invest New Drugs. 2014;32(5):783-794.

37. Liu DSH, Read M, Cullinane C, et al. APR246 potently inhibits tumour growth and overcomes chemoresistance in preclinical models of oesophageal adenocarcinoma. Gut. 2015;64(10):1506-1516.

38. Ogiwara H, Takahashi K, Sasaki M, et al. Targeting the vulnerability of glutathione metabolism in ARID1A-deficient cancers. Cancer Cell. 2019;35(2):177-190.e8.

39. Ali D, Jönsson-Videsäter K, Deneberg S, et al. APR-246 exhibits anti-leukemic activity and synergism with conventional chemotherapeutic drugs in acute myeloid leukemia cells. Eur J Haematol. 2011;86 (3):206-215.

40. Nahi H, Lehmann S, Mollgard L, et al Effects of PRIMA-1 on chronic lymphocytic leukaemia cells with and without hemizygous p53 deletion. Br J Haematol. 2004; 127(3):285-291.

41. Miess $\mathrm{H}$, Dankworth B, Gouw AM, et al. The glutathione redox system is essential to prevent ferroptosis caused by impaired lipid metabolism in clear cell renal cell carcinoma. Oncogene. 2018;37(40):5435-5450

42. Tsoi J, Robert L, Paraiso K, et al. Multi-stage differentiation defines melanoma subtypes with differential vulnerability to drug induced iron-dependent oxidative stress. Cancer Cell. 2018;33(5):890-904.e5.

43. Zou Y, Palte MJ, Deik AA, et al. A GPX4dependent cancer cell state underlies the clear-cell morphology and confers sensitivity to ferroptosis. Nat Commun. 2019;10(1):1617.

44. Yu Y, Xie Y, Cao L, et al. The ferroptosis inducer erastin enhances sensitivity of acute myeloid leukemia cells to chemotherapeutic agents. Mol Cell Oncol. 2015; 2(4):e1054549.

45. Jones CL, Stevens BM, D'Alessandro A, et al. Cysteine depletion targets leukemia stem cells through inhibition of electron transport complex II. Blood. 2019;134(4): 389-394.

46. Hoeks M, Yu G, Langemeijer S, et al. Impact of treatment with iron chelation therapy in patients with lower-risk myelodysplastic syndromes participating in the European MDS registry. Haematologica. 2020;105(3):640-651.

47. Wu J-R, Tuo Q-Z, Lei P. Ferroptosis, a recent defined form of critical cell death in neurological disorders. J Mol Neurosci. 2018;66(2):197-206

48. Cardoso BR, Hare DJ, Bush AI, Roberts BR. Glutathione peroxidase 4: a new player in neurodegeneration? Mol Psychiatry. 2017; 22(3):328-335. 